\title{
COMPORTAMIENTO DE EDIFICIOS DE ACERO CON CONTRAVENTEOS EXCÉNTRICOS ANTE SECUENCIAS SÍSMICAS
}

\author{
Jorge Ruiz García ${ }^{(1)}$, Edén Bojórquez Mora ${ }^{(2)}$, Edgar Corona Villar ${ }^{(3)}$,Alfredo Reyes Salazar ${ }^{(2)}$
}

\begin{abstract}
RESUMEN
En este artículo se presentan los resultados de un estudio analítico enfocado a evaluar el comportamiento sísmico de edificios de acero estructurados a base de marcos y contraventeos excéntricos (MCE). Para tal fin, se modelaron dos MCE de 4 y 8 niveles mediante la plataforma computacional OpenSees. En particular, se modeló el comportamiento histerético de los eslabones en los contraventeos excéntricos considerando un criterio de falla, así como la zona del panel en las uniones viga-columna. Los MCE en estudio fueron sometidos a un conjunto de secuencias sísmicas evento principal-réplicas artificiales, las cuales son representativas de secuencias registradas en terreno blando de la Ciudad de México. Los resultados de está investigación indican que los eslabones pueden fallar ante la acción de réplicas intensas, lo cual conduce a un incremento de sus demandas de distorsión máxima de entrepiso respecto a las originadas por el evento principal. Ante esta situación, se observó que las vigas y columnas cercanas al eslabón que falla exhiben un comportamiento inelástico, lo cual es contrario a su filosofía de diseño. Finalmente, se aprecio que los MCE exhiben una concentración de distorsión máxima de entrepiso en los pisos inferiores dado que los eslabones no tienen un comportamiento histerético uniforme en la altura, lo cual desaprovecha su capacidad de disipación de energía.
\end{abstract}

Palabras Clave: contraventeos excéntricos; eslabón; secuencias sísmicas; distorsión máxima de entrepiso

\section{BEHAVIOR OF STEEL BUILDINGS WITH ECCENTRICALLY BRACED FRAMES SUBJECTED TO SEISMIC SEQUENCES}

\begin{abstract}
This paper presents the results of an analytical study focused on evaluating the seismic behavior of steel buildings having eccentric braced frames (EBFs) as structural system. For this purpose, two 4story and 8-story EBFs were modeled using the computational platform OpenSees. Particularly, modelling strategy included the hysteretic behavior of the links in the eccentric braces taking into account a failure criterion as well as the panel zone in the beam-column joints. The case-study EBFs were subjected to a set of artificial mainshock-aftershock seismic sequences, which are representative
\end{abstract}

\footnotetext{
Artículo recibido el 25 de agosto de 2017 y aprobado para su publicación el 2 de julio de 2019. Se aceptarán comentarios y/o discusiones hasta cinco meses después de su publicación.

(1) Universidad Michoacana de San Nicolás de Hidalgo. Cd. Universitaria, Av. Francisco J. Mújica S/N, Morelia 58040. jruizgar@stanfordalumni.org

(2) Facultad de Ingeniería Culiacán, Universidad Autónoma de Sinaloa, Calzada de las Américas y Blvd. Universitarios, Culiacán, 80040. eden_bmseg@hotmail.com, reyes@uas.edu.mx

(3) Egresado de la Maestría en Ingeniería, Facultad de Ingeniería Culiacán, Universidad Autónoma de Sinaloa, Calzada de las Américas y Blvd. Universitarios, Culiacán 80040. edgar_corvi@ hotmail.com
} 
of those recorded in soft soil sites of Mexico City. The results of this investigation reveal that the links can fail under strong aftershocks, which lead to an increment in the maximum interstory drifts with respect to those from the mainshock. In this situation, it was observed that the beams and columns near the link that failed can exhibit nonlinear behavior, which is opposite to its design philosophy. Finally, it was also noted that the EBFs tends to concentrate the maximum interstory drifts at the bottom stories since the links do not exhibit a uniform hysteretic behavior along height, which does not take fully advantaje of the energy dissipation capacity of the links.

Keywords: seismic sequences; eccentrically braced frames; steel frames; seismic response

\section{INTRODUCCIÓN}

Existe un creciente interés por incorporar marcos con contraventeos excéntricos (MCE) como sistema estructural sismorresistente para edificios en la Ciudad de México, y en otras regiones del país, como se ilustra en la figura 1, lo cual puede atribuirse a que aportan tanto una alta rigidez elástica (similar a los contraventeos concéntricos) como ductilidad (similar a los marcos resistentes a momento). En los MCE, la energía inducida durante un evento sísmico es disipada por el comportamiento inelástico de los eslabones, mientras que las vigas, columnas y contraventeos se diseñan para exhibir un comportamiento elástico. Este sistema estructural fue propuesto en Japón en los años 70's y fue investigado extensamente en los Estados Unidos por Popov y sus colaboradores en los años 80's (Azad y Topkaya, 2017). Una revisión detallada de los estudios experimentales y analíticos más relevantes sobre el desarrollo de contraventeos excéntricos puede consultarse en (Azad y Topkaya, 2017).

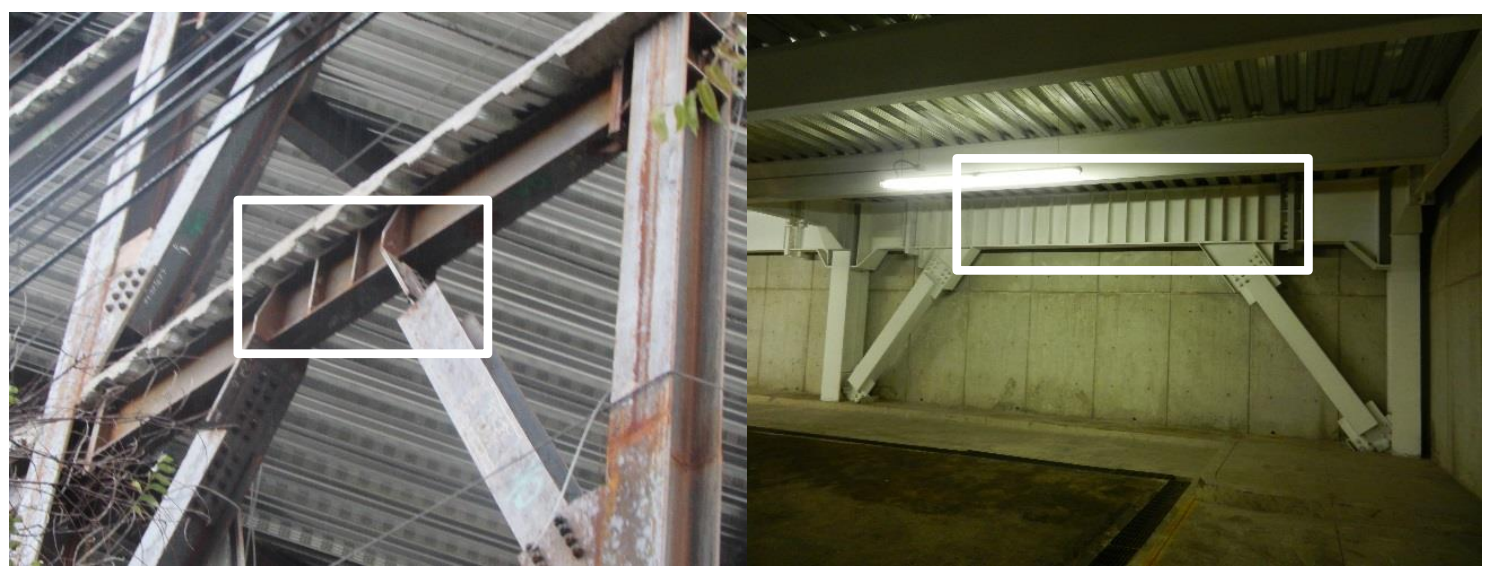

(a)

(b)

Figura 1. Ejemplos del uso de contraventeos excéntricos en México con diferentes tipos de eslabón (indicado en cuadro blanco, Fotos del primer autor): a) eslabón corto, b) eslabón largo

Para fines de diseño, las Normas ASCE 7-88 (1990) permitieron el uso de MCE en los Estados Unidos como un sistema estructural de alta ductilidad. Posteriormente, con base en extensa investigación experimental, las especificaciones ANSI/AISC 341 del Instituto Americano de la Construcción en Acero incluyeron un procedimiento de diseño detallado de los eslabones, siendo la versión ANSI/AISC 341-16 la más actualizada (ASCE, 2016). En ella se especifican el tipo de eslabón (corto, intermedio, o largo), las rotaciones permisibles del eslabón, la sobrerresistencia que se puede considerar, entre otros aspectos. Particularmente, la rotación del eslabón se limita a 0.08 radianes para eslabones con comportamiento a cortante (es decir, para eslabones con longitud, $e$, menor o igual a $1.6 M_{p} / V_{p}$, donde $M_{p}$ y $V_{p}$ son el momento plástico y la resistencia a cortante plástica del eslabón), denominados eslabones cortos, y a 0.02 radianes 
para eslabones con comportamiento a flexión (es decir, para eslabones con longitud mayor o igual a $2.6 \mathrm{M}_{\mathrm{p}} / \mathrm{V}_{\mathrm{p}}$ ), denominados eslabones largos. Para eslabones con longitud, $e$, entre $1.6 M_{p} / V_{p}$ y $2.6 M_{p} / V_{p}$, la rotación límitrofe se obtiene por interpolación lineal. Sin embargo, no obstante el interés por este sistema estructural entre la comunidad ingenieril mexicana, hasta la reciente edición 2017 de la Normas Técnicas Complementarias para el Diseño y Construcción de Estructuras de Acero (NTC-A, 2017) se proporcionan lineamientos para su diseño, los cuales se basan en las especificaciones ANSI/AISC 341-16.

Las estructuras ubicadas en zonas de alto peligro sísmico no son expuestas a un solo evento, sino a una secuencia sísmica conformada por precursores-evento principal-réplicas. En este ambiente sísmico, las réplicas pueden inducir demandas de distorsión lateral mayores a aquellas inducidas ante el evento principal. Como consecuencia, las réplicas pueden incrementar el estado de daño observado después del evento principal o, inclusive, ocasionar la necesidad de demoler la estructura si se incrementan sus desplazamientos permanentes al final del evento principal. Un claro ejemplo de este escenario ocurrió durante el sismo del 19 de septiembre de $1985\left(\mathrm{M}_{\mathrm{w}}=8.0\right)$ y la subsecuente réplica del 20 de septiembre de $1985\left(\mathrm{M}_{\mathrm{w}}=7.6\right)$ que afectaron significativamente a la Ciudad de México. Inspecciones de campo llevadas a cabo después del evento principal, corroborados con estudios analíticos, evidenciaron que las estructuras de mediana altura, principalmente estructuradas a base de marcos de concreto reforzado (CR) de entre 8 y 18 niveles, sufrieron daño moderado a grave (Rosenblueth y Meli, 1986; Meli y Ávila, 1989). A consecuencia de la réplica ocurrida al día siguiente, varias estructuras incrementaron su estado de daño, o terminaron con desplazamientos laterales permanentes excesivos al final de la excitación. No obstante este hecho, muy pocas investigaciones se han desarrollado para investigar el efecto de las réplicas en la respuesta de estructuras desplantadas en terreno blando (Ruiz-García et al., 2014; Díaz-Martínez et al., 2014; Guerrero et al., 2017). Otro ejemplo de la acción de réplicas intensas se pudo observar durante la secuencia sísmica evento principal-réplicas ocurrida en Nueva Zelanda en los años 2010-2011, dado que el evento principal ocurrió el 4 de septiembre de $2010\left(\mathrm{M}_{\mathrm{w}}=7.1\right) \mathrm{y}$, posteriormente, ocurrieron una serie de réplicas intensas, siendo la más intensa la ocurrida el 22 de febrero de $2011\left(\mathrm{M}_{\mathrm{w}}=6.3\right)$. A consecuencia del evento del 22 de febrero, se pudo observar un comportamiento inadecuado en los contraventeos excéntricos y falla de algunos eslabones de una estructura para estacionamiento ubicada en la ciudad de Christchurch (Kanvinde et al., 2015).

Cabe mencionar que existe evidencia de que la intensidad de las réplicas, medida por la aceleración máxima del terreno registrada en una estación acelerográfica, puede ser mayor que la debida al evento principal. Por ejemplo, las estaciones Papanoa y Aeropuerto Zihuatanejo registraron mayor aceleración máxima del terreno durante la réplica del 20 de septiembre de 1985. Asimismo, varias estaciones acelerográficas en la ciudad de Christchurch, Nueva Zelanda, registraron aceleraciones máximas del terreno sensiblemente mayores durante el evento del 22 de febrero de 2011 que aquellas durante el evento principal del 4 de septiembre de 2010. Lo anterior puede atribuirse a que las réplicas pueden tener una menor distancia epicentral que el evento principal a un sitio dado, aun cuando tengan menor magnitud (Ruiz-García, 2012).

El objetivo principal de esta investigación consistió en investigar el comportamiento sísmico de dos marcos de acero con contraventeos excéntricos ubicados en la zona de terreno blando de la Ciudad de México al ser sujetos a secuencias sísmicas evento principal-réplica. Para tal fin, se modelaron y analizaron dos marcos de 4 y 8 niveles, prestando especial atención al modelado de los eslabones, el cual incluye un criterio de falla. A partir de está investigación, se discute el comportamiento de los edificios estudiados y sus implicaciones en la práctica profesional mexicana. Sin embargo, debe notarse que los resultados y conclusiones ofrecidas en este artículo pueden variar respecto a otros edificios con diferente configuración de contraventeos excéntricos y número de niveles. 


\section{EDIFICIOS CONSIDERADOS EN ESTA INVESTIGACIÓN}

\section{Descripción}

Para fines de esta investigación, se seleccionaron edificios de acero de 4 y 8 niveles que son representativos de edificaciones de baja y mediana altura. Los edificios fueron diseñados por Díaz (2006), bajo la supervisión de un despacho profesional, considerando la edición 2004 del Reglamento de Construcciones del Distrito Federal y sus correspondientes Normas Técnicas Complementarias para el Diseño Sísmico (NTC-S, 2004), así como para el Diseño y Construcción de Estructuras Metálicas (NTC-A, 2004). Ambos edificios están estructurados a base de marcos resistentes a momento en una dirección y marcos con contraventeos excéntricos (MCE) en la dirección ortogonal. En la figura 2 se muestra la vista en planta de los edificios estudiados, mientras que en la figura 3 se presenta la elevación de los marcos que incluyen contraventeos excéntricos. El uso de los edificios es para oficinas y se consideró que estaban ubicados en la Zona IIIb de la Ciudad de México. En su diseño se consideró un factor de comportamiento sísmico, $Q$, igual a 3 . Las vigas y columnas fueron diseñados considerando acero A-36, con una resistencia nominal de fluencia $F_{y}=2530 \mathrm{~kg} / \mathrm{cm}^{2}$, mientras que para los contraventeos se considero acero A-50, con una resistencia nominal de fluencia $F_{y}=3515 \mathrm{~kg} / \mathrm{cm}^{2}$. Se especificó que los eslabones de los MCE tendrían una longitud igual al 20\% de la longitud del claro del marco. En la tabla 1 se indican las secciones finales de los contraventeos y eslabornes para ambos marcos. Una descripción detallada del proceso de diseño de los edificios puede consultarse en Díaz (2006).

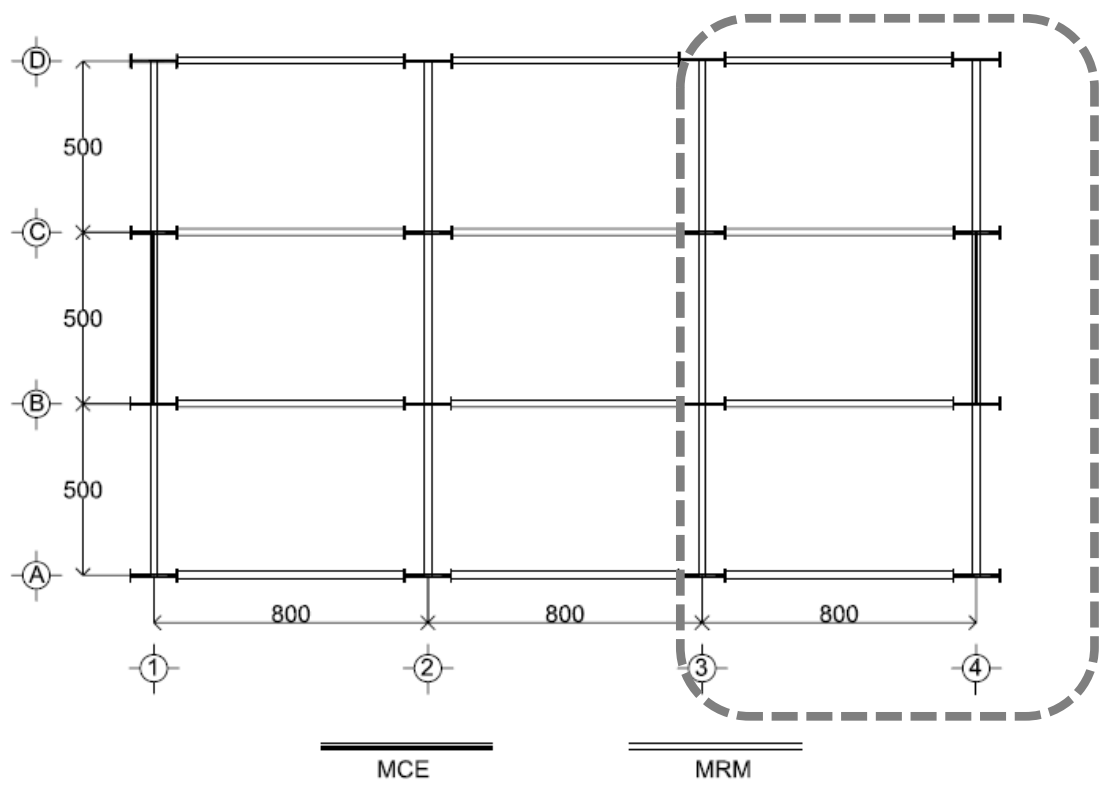

Figura 2. Planta estructural de los edificios en estudio (acotación en cm) 


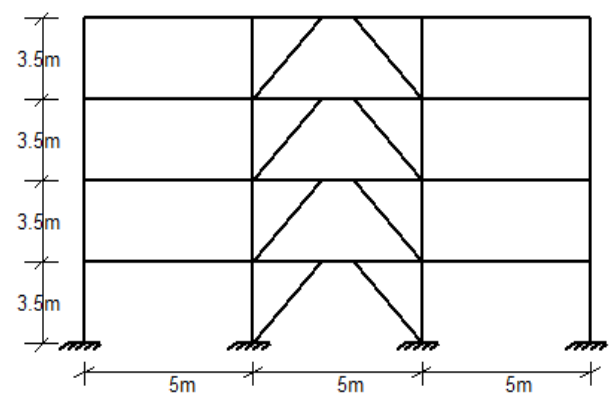

a)

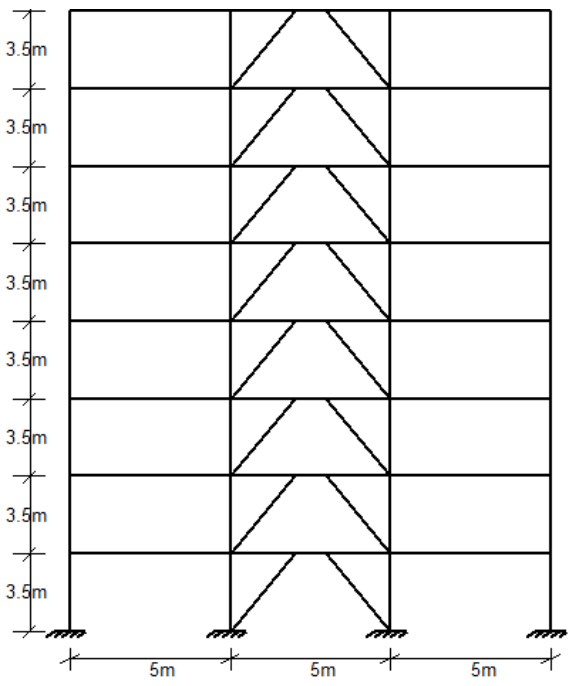

b)

Figura 3. Elevación de los marcos con contraventeos excéntricos (acotación en m): a) 4 niveles, b) 8 niveles

Tabla 1. Secciones consideradas para el eslabón y contraventeos en los edificios de 4 y 8 niveles (Díaz, 2004)

\begin{tabular}{|c|c|c|c|c|}
\hline Nivel & Eslabón & Contraventeo & Eslabón & Contraventeo \\
\hline 8 & - & - & IR457x74.5 & OR203x47.38 \\
\hline 7 & - & - & IR457x74.5 & OR203x47.38 \\
\hline 6 & - & - & IR457x89.1 & OR $203 \times 47.38$ \\
\hline 5 & - & - & IR457x96.7 & OR203x47.38 \\
\hline 4 & IR406x59.8 & OR203x47.38 & IR553x108.9 & OR203x47.38 \\
\hline 3 & IR406x67.4 & OR203x47.38 & IR553x108.9 & OR203x47.38 \\
\hline 2 & IR406x85.1 & OR203x47.38 & IR553x123.1 & OR203×56.10 \\
\hline 1 & IR406x99.8 & OR203x47.38 & IR553x 123.1 & OR203×56.10 \\
\hline
\end{tabular}

\section{Modelado}

El interés de esta investigación se centrá en el comportamiento sísmico de MCE, por lo cual sólo se modeló la dirección transversal de los edificios en estudio con el uso de la plataforma computacional OpenSees (McKenna et al., 2000). Dada la simetría en planta, sólo se modeló la mitad del edificio acoplando bidimensionalmente un marco exterior y un marco interior, los cuales estaban ligados mediante barras horizontales rígidas para simular un diafragma rígido, como se ilustra en la figura 2. Se supuso que las columnas estaban empotradas en su base, por lo que no se consideró la interacción suelo-estructura ni la flexibilidad de la base de las columnas. Las vigas, columnas y contraventeos se modelaron con un criterio de plasticidad distribuida discretizando las secciones transversales en fibras. Para modelar el comportamiento del acero se utilizó el modelo de Giuffré-Menegotto-Pinto (el cual está incluido en la biblioteca del programa OpenSees como material steel02 (McKenna et al., 2000)). Este modelo es capaz de simular el endurecimiento por deformación cinemática e isotrópica, característica que se presenta en el acero. Cabe notar que el modelo de Giuffré-Menegotto-Pinto no es capaz de simular la degradación de 
rigidez y resistencia, lo cual es una limitante del modelo; sin embargo, se espera que las vigas, columnas y contraventeos se comporten elásticamente durante la acción sísmica de acuerdo con su filosofía de diseño.

La zona de panel se presenta en las intersecciones de los elementos columna y viga. En esta región, que está delimitada por los peraltes de la columna y viga, se presenta un estado de esfuerzos y deformaciones complejo debido a la transferencia de momentos entre los elementos. La zona de panel se deforma principalmente por cortante debido a los momentos opuestos en vigas y columnas. Para considerar el efecto de zona de panel, en este estudio se incluyó el modelo propuesto por Krawinkler (Gupta y Krawinkler, 1999). El modelo considera un rectángulo conformado por elementos rígidos, los cuales no se deformarán, y un resorte rotacional el cual considera el comportamiento histerético debido a cortante. En la figura 4a se muestra la estrategia de modelado de la zona de panel, mientras que la envolvente cortante-deformación considerada en este estudio se muestra en la figura $4 \mathrm{~b}$.

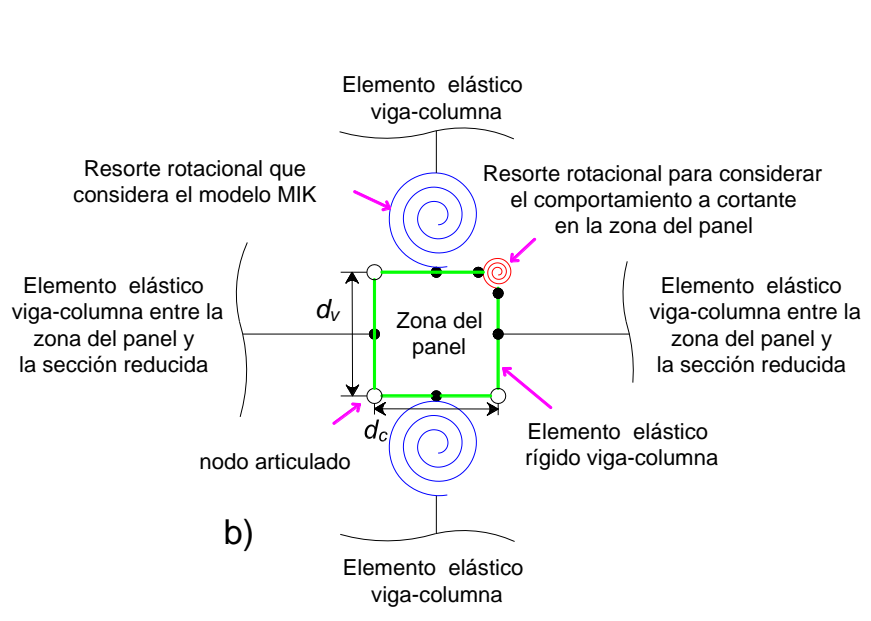

(a)

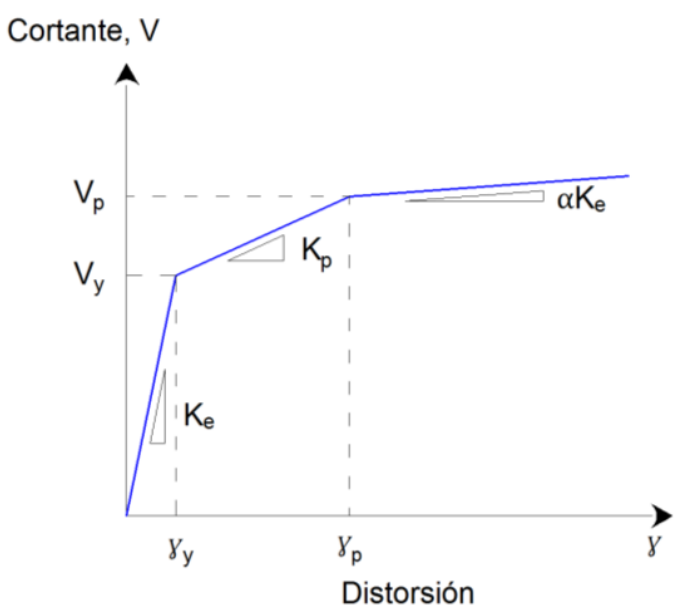

(b)

Figura 4. a) Modelo analítico utilizado para representar el efecto de la zona de panel, b) Relación fuerza-deformación a cortante para la zona de panel

Para la modelación de los eslabones se empleó la técnica utilizada por Richards (2004) y Prinz (2010), la cual es una versión modificada de la propuesta por Ramadan y Ghobarah (1995). Esta técnica considera que los eslabones se modelan como una viga elástica con plasticidad distribuida en los extremos del elemento (definida por el elemento forceBeamColumn, anteriormente llamado beamwithHinges, en la biblioteca de OpenSees), así como cuatro resortes traslacionales actuando en paralelo para representar una articulación por cortante en los extremos como se ilustra en la figura 5a. En el modelo analítico del eslabón ilustrado en la figura 5a, el elemento elástico no considera las deformaciones por cortante (al indicar un área de cortante nula) y, en consecuencia, se desprecia la rigidez a cortante. Para simular el comportamiento fuerza cortante-deformación en el extremo de cada uno de los eslabones, a cada resorte en la articulación a cortante se le asignó un comportamiento elastoplástico y se consideró que actuaban en paralelo para obtener un nuevo material (mediante el comando uniaxialMaterial Parallel, en la biblioteca de OpenSees). Este material tiene un comportamiento ilustrado en la figura $5 \mathrm{~b}$, el cual se le asignó a un elemento de longitud cero (elemento zeroLength en la biblioteca de OpenSees) definido por dos nodos, interno y externo, con la misma ubicación y el mismo desplazamiento horizontal. Cabe notar que la rigidez inicial de cada articulación a cortante, $K v_{1}$, es igual a $2 G A_{\text {corte }} / e$ (donde $G, A_{\text {corte }}$ y $e$ son el modulo de cortante, el área de cortante de la sección y la longitud del eslabón, respectivamente) dado que ambas articulaciones actúan en serie, dando como resultado la rigidez del eslabón igual a $G A_{\text {cortele }}$ (Richards, 2004). Para fines de análisis, 
la rotación del eslabón se calculó como el desplazamento vertical entre ambos nodos externos dividido entre la longitud del eslabón.

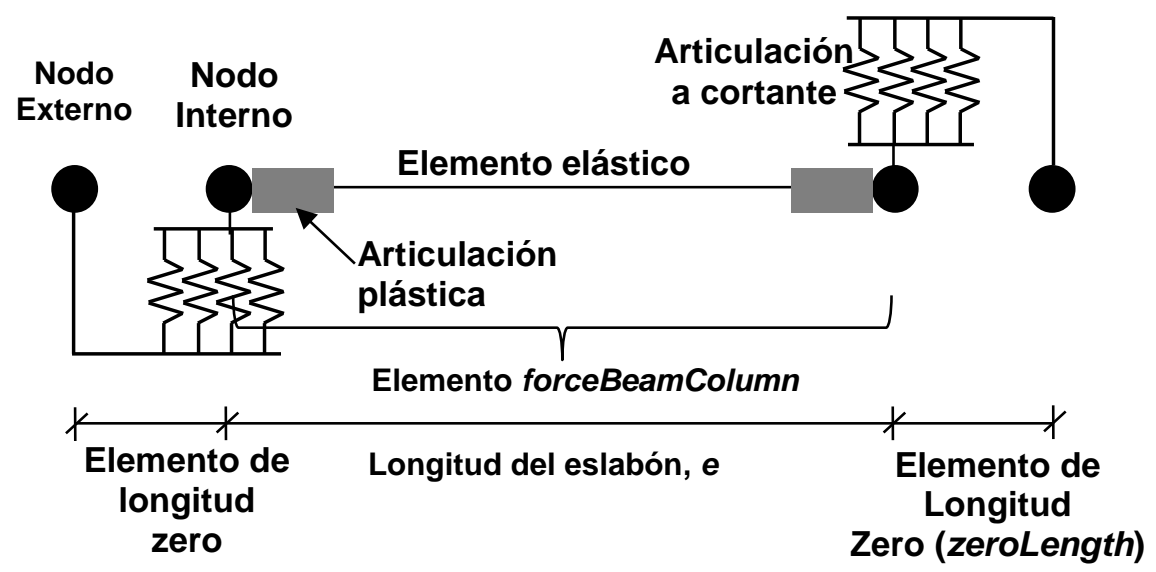

(a)

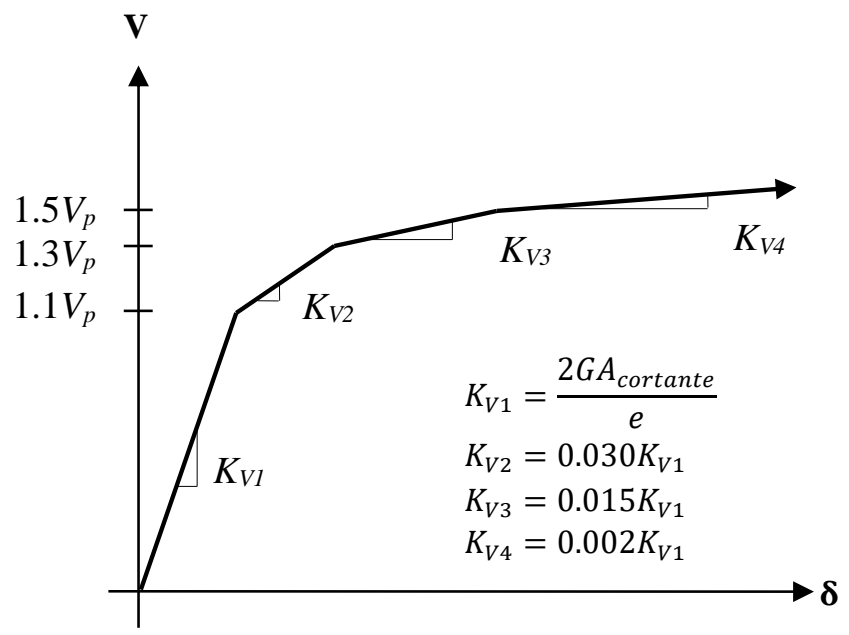

(b)

Figura 5. a) Modelo analítico para simular el comportamiento histerético del eslabón (Richards, 2004),

b) Envolvente del comportamiento fuerza cortante-deformación en cada articulación a cortante

Cabe notar que en el diseño de los elementos de los edificios en estudio se utilizaron valores nominales del esfuerzo de fluencia. Para tener en cuenta la diferencia entre los valores nominales y los esperados, en esta investigación se consideró un esfuerzo de fluencia esperado de $3,460 \mathrm{~kg} / \mathrm{cm}^{2}$ y 4,050 $\mathrm{kg} / \mathrm{cm}^{2}$ para el acero A-36 y el acero A-50 (Gupta y Krawinkler, 1999), respectivamente, para calcular la resistencia a flexión y cortante de las secciones transversales en vigas, columnas, contraventeos, zona del panel y los eslabones. Investigaciones recientes sobre la calidad del acero en perfiles estructurales y placas comúnmente fabricados en el mercado mexicano mostró que los perfiles IR de acero A572 Gr. 50 y perfiles OR de acero A500 Grado B tenían esfuerzos de fluencia medios de 4,223 kg/cm y 3,995 kg/cm respectivamente, mientras que sus esfuerzos de fluencia nominales eran de $3,515 \mathrm{~kg} / \mathrm{cm}^{2}$ y $3,235 \mathrm{~kg} / \mathrm{cm}^{2}$, respectivamente (Tapia y Tena, 2011). Por ello, se consideran razonables los esfuerzos de fluencia considerados en este estudio para considerar la sobrerresistencia debido al esfuerzo de fluencia del acero estructural. 
Para fines de esta investigación, se denominó a los modelos analíticos como 4N_MCE y 8N_MCE. Una vez terminada su modelación, se realizó un análisis modal convencional para calcular los periodos fundamentales de vibración de cada modelo. Los periodos de vibración correspondientes al primer y segundo modos de vibración del modelo 4N_MCE fueron de $0.77 \mathrm{seg}$. y $0.29 \mathrm{seg}$, respectivamente, mientras que para el modelo $8 \mathrm{~N} \_\mathrm{MCE}$ fueron de $1.32 \mathrm{seg}$. y $0.48 \mathrm{seg}$., correspondientes al primer y segundo modo de vibración.

\section{Respuesta analítica y criterio de falla del eslabón}

Para verficar el comportamiento histerético descrito por el modelo analítico del eslabón, se simuló en la plataforma computacional OpenSees el dispositivo de ensaye empleado por Okazaki et al. (2005, 2007), el cual reproduce el entorno de fuerzas y deformaciones a los que está sometido un eslabón de un marco de baja altura, que se encuentra unido en un extremo a una columna y en el otro al contraventeo como se muestra la figura 6a. La cinemática del dispositivo de ensaye y la ubicación del especimen del eslabón se muestran en la figura $6 \mathrm{~b}$.

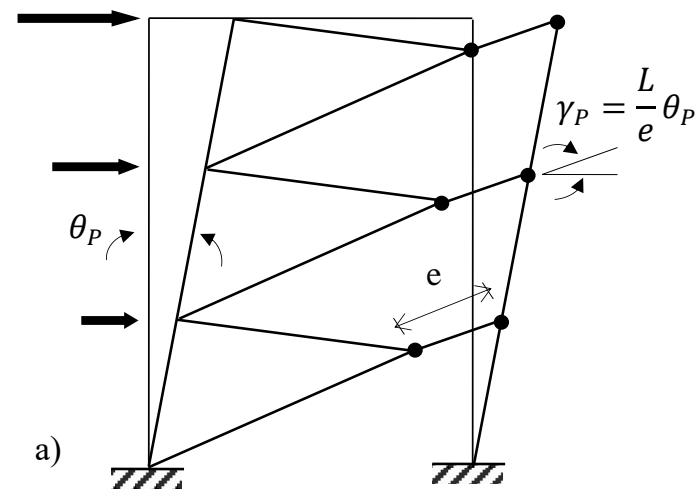

b)

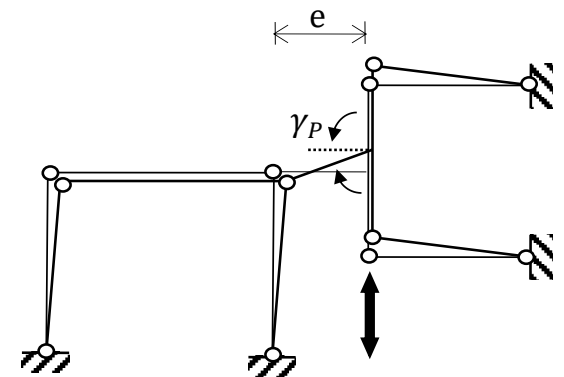

Figura 6. a) Mecanismo de falla de un marco típico con contraventeo excéntrico, b) Dispositivo de ensaye de un eslabón utilizado por Okazaki et al. $(2005,2007)$

Empleando este dispositivo de ensaye, Okazaki et al. (2005, 2007) ensayaron 37 especímenes de eslabones con diferentes características geométricas. En la Figura 7 se muestra la respuesta histerética fuerza cortante-rotación medida en los especímenes $4 \mathrm{~A}$ y 5 . En su estudio, los autores observaron consistentemente la fractura del alma en eslabones con longitud $e<1.7 M_{p} / V_{p}$ lo cual limitó la capacidad de rotación inelástica a valores menores que 0.08 rad como lo indicaba la normatividad (AISC, 2002). La fractura prematura del alma también se reflejó en una degradación de resistencia severa en el comportamiento histerético, como se aprecia en la figura 7, a partir de rotaciones cercanas a 0.06 rad (línea punteada rosa). Por ello, en está investigación se decidió incorporar un criterio de falla en la respuesta histerética del eslabón, el cual consiste en asumir que el eslabón agota su capacidad a fuerza cortante una vez que alcanza una rotación igual a 0.06 rad. En la figura 8 se muestra la respuesta histerética analítica del espécimen 4A (Okazaki et al. 2005) obtenida con la estrategía de modelado del eslabón, propuesta en Richards (2004), e incorporada en esta investigación, la cual reproduce razonablemente bien la respuesta experimental.

\section{SECUENCIAS SÍSMICAS}

Una revisión de las historias de aceleración incluidas en la Base Mexicana de Datos de Sismos Fuertes (SMIS, 1999) evidenció que sólo se encuentra disponible una secuencia sísmica evento principal-réplica registrada en terreno blando, la cual se registró en la estación Central de Abastos (CDAF) durante los sismos 
ocurridos el 19 y 20 de septiembre de 1985 (Ruiz-García et al., 2014). En la figura 9 se presentan las historias de aceleración registradas en dicha estación, e información relevante se indica en la tabla 2. En este estudio, el periodo predominante de la excitación, $T_{g}$, se determinó como el periodo de vibración asociado a la ordenada máxima en el espectro elástico de velocidad (Miranda, 1993).

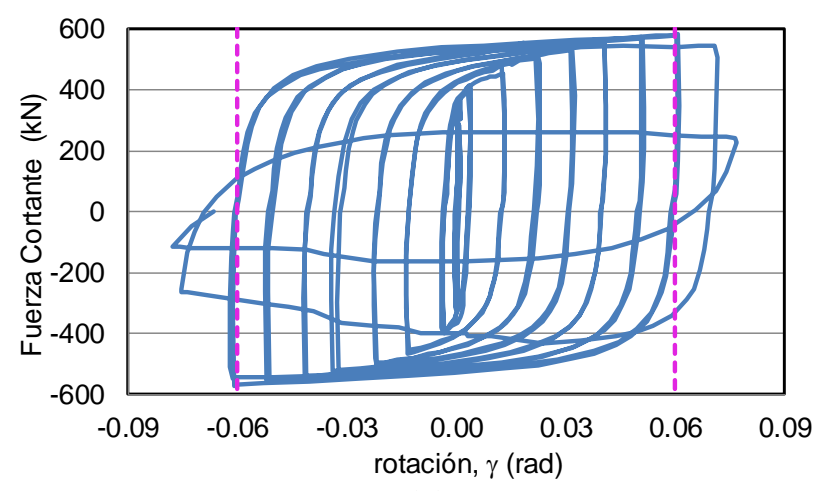

(a)

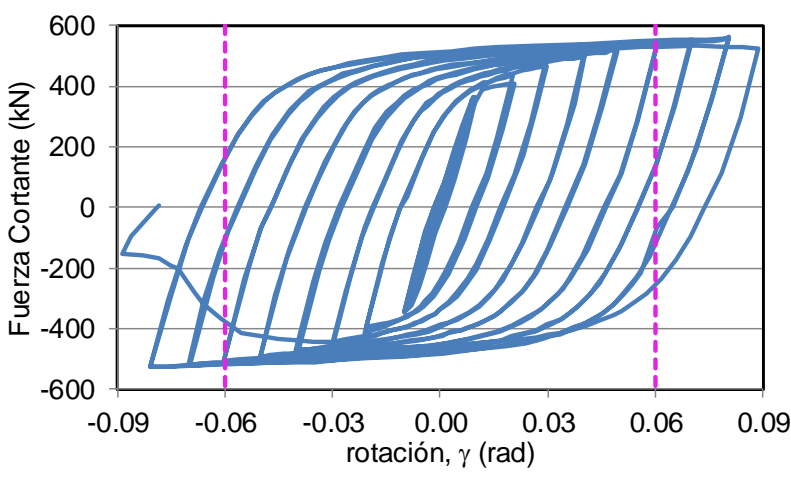

(b)

Figura 7. Comportamiento histerético del: a) espécimen 4A (Okazaki y Engelhardt, 2009) y b) espécimen 5 (Okazaki y Engelhardt, 2009)

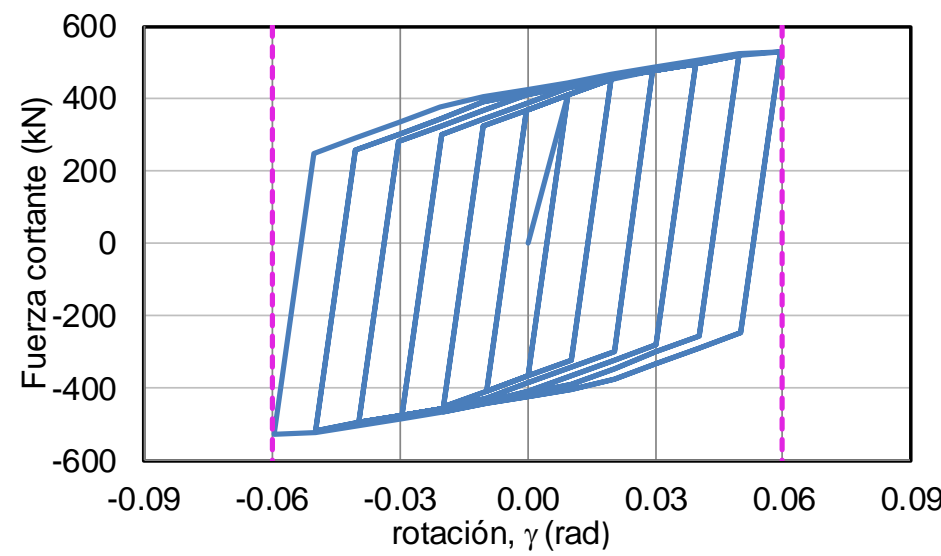

Figura 8. Respuesta histerética analítica del espécimen 4A obtenida con la estrategía de modelado del eslabón propuesta en Richards (2004), e incorporada en esta investigación

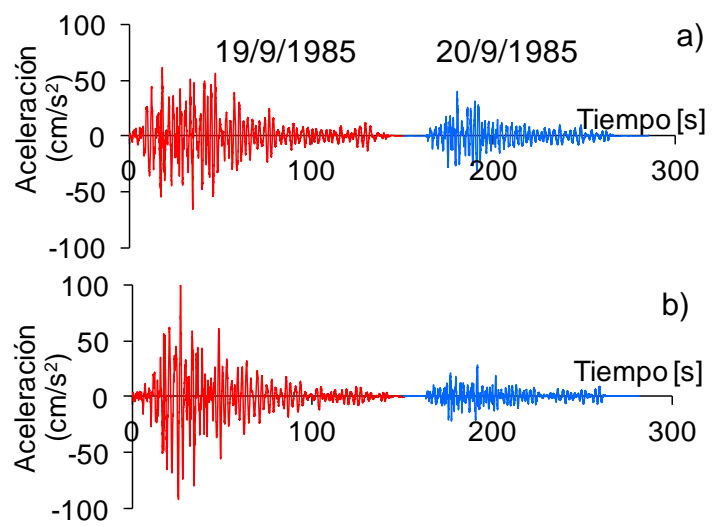

Figura 9. Secuencias evento principal-réplica registradas en la estación CDAF durante los sismos de septiembre de 1985: a) componente NO0E, b) componente N90E 
Tabla 2. Características de las secuencias sísmicas registradas en terreno blando de acuerdo a la Base Mexicana de Datos de Sismos Fuertes (SMIS, 1999)

\begin{tabular}{|c|c|c|c|c|c|c|c|}
\hline \multirow[b]{3}{*}{ Fecha } & \multirow[b]{3}{*}{ Ms } & \multicolumn{3}{|c|}{ Estación } & \multirow{3}{*}{$\frac{\text { AMT }}{\left(\mathrm{cm} / \mathrm{s}^{2}\right)}$} & \multirow{3}{*}{$\frac{\mathrm{VMT}}{(\mathrm{cm} / \mathrm{s})}$} & \multirow{3}{*}{$\frac{\mathrm{T}_{\mathrm{g}}}{\text { (seg.) }}$} \\
\hline & & Nombre de & ID & Comp. & & & \\
\hline & & la estación & & & & & \\
\hline $19 / 09 / 1985$ & 8.1 & $\begin{array}{c}\text { Central de } \\
\text { Abastos }\end{array}$ & CDAF & NOOE & 66.3 & 26.4 & 2.88 \\
\hline 19/09/1985 & 8.1 & $\begin{array}{c}\text { Central de } \\
\text { Abastos }\end{array}$ & CDAF & N90E & 95.9 & 36.7 & 2.96 \\
\hline 20/09/1985 & 7.6 & $\begin{array}{c}\text { Central de } \\
\text { Abastos }\end{array}$ & CDAF & NOOE & 40.1 & 12.1 & 2.28 \\
\hline 20/09/1985 & 7.6 & $\begin{array}{c}\text { Central de } \\
\text { Abastos }\end{array}$ & CDAF & N90E & 30.2 & 9.3 & 3.01 \\
\hline
\end{tabular}

Ante la ausencia de secuencias sísmicas reales (es decir, registradas en estaciones acelerográficas), como parte de este estudio se generaron secuencias sísmicas artificiales a partir de la combinación aleatoria de acelerogramas reales registrados en terreno blando durante eventos sísmicos históricos. Para ello, se consideraron 8 historias de aceleración registradas en estaciones ubicadas en terreno blando de la Ciudad de México durante eventos principales. En la tabla 3 se indican las características de los 8 registros.

Tabla 3. Lista de las historias de aceleración empleadas para generar secuencias sísmicas artificiales

\begin{tabular}{cccccccc}
\hline$\#$ & Fecha & Ms & $\begin{array}{c}\text { No. } \\
\text { Estación }\end{array}$ & Nombre & Comp. & $\begin{array}{c}\text { AMT } \\
\left(\mathrm{cm} / \mathrm{s}^{2}\right)\end{array}$ & $\begin{array}{c}\mathrm{T}_{\mathrm{g}} \\
(\mathrm{s})\end{array}$ \\
\hline 1 & $25 / 04 / 1989$ & 6.9 & 29 & Villa del mar & EW & 46.5 & 2.96 \\
2 & $25 / 04 / 1989$ & 6.9 & 29 & Villa del mar & NS & 49.4 & 2.96 \\
3 & $25 / 04 / 1989$ & 6.9 & 43 & Jamaica & NS & 35.2 & 3.04 \\
4 & $25 / 04 / 1989$ & 6.9 & 48 & Rodolfo Menéndez & EW & 47.7 & 2.89 \\
5 & $25 / 04 / 1989$ & 6.9 & 25 & P.C.C. Superficie & EW & 42.5 & 2.30 \\
6 & $14 / 09 / 1995$ & 7.1 & 56 & Córdova & EW & 19.4 & 2.30 \\
7 & $25 / 04 / 1989$ & 6.9 & 58 & Liverpool & EW & 40.0 & 2.30 \\
8 & $14 / 09 / 1995$ & 7.1 & RB & Roma-B & EW & 25.0 & 2.30 \\
\hline
\end{tabular}

Para la generación de las secuencias artificiales, se empleó el criterio aleatorio, descrito en RuizGarcía (2012), el cual consiste en seleccionar un registro como evento principal y los restantes registros se emplean como 'réplicas', sin repetición de eventos. Cabe notar que sólo los registros con $T_{g}$ cercano a 3.0 seg. se emplearon como eventos principales (del \#1 al \#4 en la tabla 3), con la finalidad de generar secuencias sísmicas artificiales con contenido de frecuencia similar en el evento principal y en la réplica al observado en las secuencias registradas en la estación CDAF. Como medida de intensidad sísmica de las secuencias sísmicas se escogió a la velocidad máxima del terreno, VMT, dado que está altamente correlacionada con las demandas de energía impuestas a estructuras ubicadas en terreno blando (por ejemplo, Terán-Gilmore et al., 2010). La VMT más alta registrada durante eventos históricos corresponde a la registrada en la estación SCT durante el sismo del 19 de septiembre de 1985, la cual fue de aproximadamente de $61 \mathrm{~cm} / \mathrm{s}$. Por ello, antes de generar las secuencias artificiales, cada evento principal se escaló, en amplitud, para alcanzar dicha VMT. Así, para cada secuencia artifical evento principal-réplica se consideraron tres relaciones de la velocidad máxima del terreno de la réplica respecto a la velocidad máxima del terreno del evento principal, $V_{R} / V_{E P}$, iguales a $1.00,0.70$ y 0.35 . Es decir, las secuencias del primer conjunto se conformaron con eventos principales y réplicas escaladas al $100 \%$ de la velocidad máxima, $V_{E P}$, 
denominado como conjunto EP-R100\%. Las secuencias del segundo conjunto, se han formado con eventos principales escalados al 100\% y réplicas escaladas al 70\% de la velocidad máxima, denominado como conjunto EP-R70\%; mientras que en las secuencias del tercer conjunto se incluyeron eventos principales escalados al $100 \%$ y réplicas escaladas al $35 \%$ de $V_{E P}$, denominado conjunto EP-R35\%. En total se consideraron tres conjuntos de 28 secuencias sísmicas artificiales. Cabe aclarar que no obstante que las réplicas artificiales fueron escaladas para alcanzar un porcentaje de la VMT, se observó que su aceleración máxima del terreno puede ser mayor que la correspondiente al evento principal para los casos de EP- R70\% y EP-R100\% una vez que las historias de velocidad escaladas se sometieron al proceso de derivación numérica para obtener las historias de aceleración. En la figura 10 se muestran dos ejemplos de las secuencias sísmicas artificiales correspondientes a tres relaciones $V_{R} / V_{E P}$, con la misma historia de aceleración del terreno del evento principal.
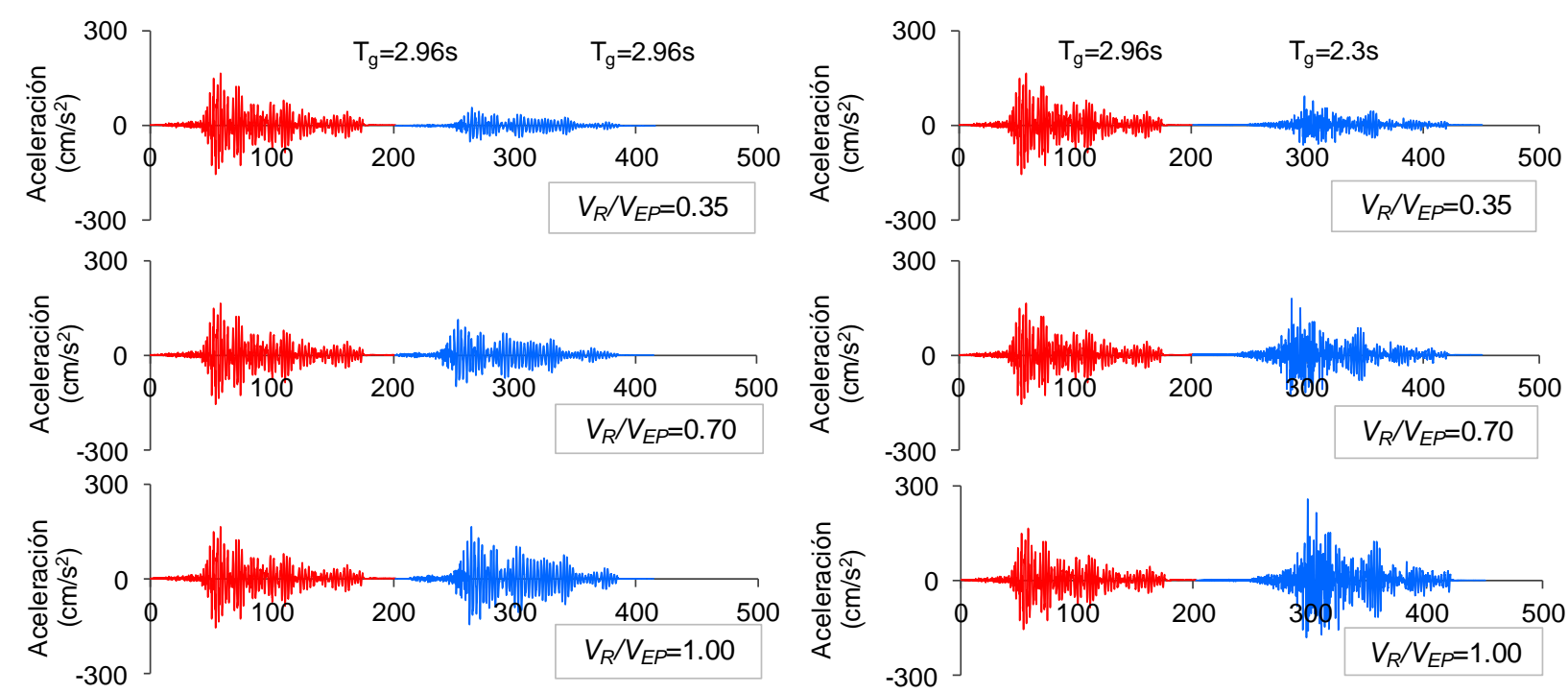

Figura 10. Ejemplos de secuencias sísmicas artificiales generadas en esta investigación empleando el mismo evento principal y diferente réplica (escaladas a tres niveles de intensidad)

En la figura 11 se ilustran los espectros de pseudo aceleración promedio correspondientes a los tres conjuntos de secuencias artificiales, así como al correspondiente al conjunto de eventos principales (EP). Se puede apreciar que los espectros de EP y EP-R35\% son idénticos; mientras que los espectros correspondientes a EP-R70\% y EP-R100\% tienen ordenadas mayores en algunos intervalos de periodo de vibración. Lo anterior puede atribuirse a que algunas historias de aceleración de las réplicas tienen aceleración máxima del terreno similar, o incluso mayor, que la aceleración máxima del terreno del evento principal como se puede observar en las secuencias del lado derecho de la figura 10, para relaciones $V_{R} / V_{E P}$ iguales a $0.70($ EP-R70\%) y $1.00(($ EP-R100\%).

\section{RESPUESTA DE LOS EDIFICIOS}

Para evaluar el desempeño sísmico de cada modelo estructural se llevó a cabo un análisis dinámico incremental descrito ampliamente en Vamvatsikos y Cornell (2002), ADI, para cada secuencia artificial. Para desarrollar el ADI se utilizó la pseudo-aceleración espectral correspondiente al modo fundamental de vibración de la estructura, $S_{a}\left(T_{1}\right)$, como medida de intensidad sísmica. De esta manera, la historia de aceleración de cada secuencia artificial evento principal-réplica se escaló, en amplitud, para alcanzar niveles 
de intensidad desde $S_{a}\left(T_{1}\right)=0.1 \mathrm{~g}$ hasta $S_{a}\left(T_{1}\right)=2.0 \mathrm{~g}$, con incrementos a cada $S_{a}\left(T_{1}\right)=0.1 \mathrm{~g}$. Sin embargo, debe notarse que los factores de escala no son los mismos para cada secuencia con un mismo nivel de $S_{a}\left(T_{1}\right)$ dada la diferencia en ordenadas espectrales para cada conjunto, como se muestra en la figura 11. La respuesta sísmica de los edificios ante cada secuencia artificial escalada a un nivel de intensidad dado se evaluó por medio de la distorsión máxima de entrepiso.

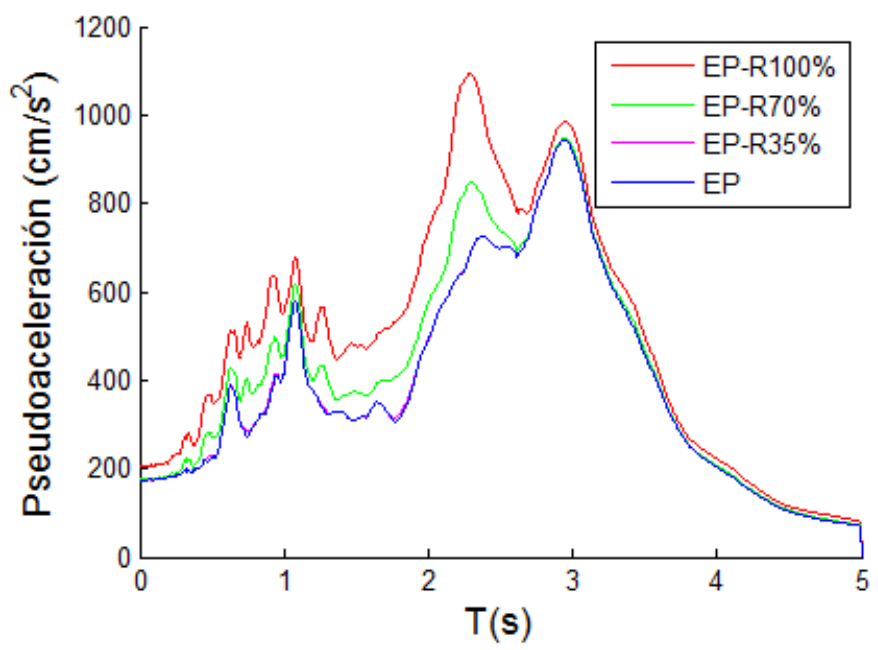

Figura 11. Comparación de los espectros de respuesta promedio correspondientes al evento principal (EP) y los tres conjuntos de secuncias sísmicas artificiales generadas en este estudio

\section{Criterio de falla del eslabón}

Con el propósito de mostrar las diferencias en incorporar o no una condición de falla en la modelación del comportamiento histerético del eslabón, se presentan los resultados de ADI's del modelo 4N_MCE sujeto a la secuencia EP2R6(70\%) (es decir, se utilizan los registros 2 y 6 de la tabla 3 como evento principal y réplica, respectivamente, considerando una relación $V_{R} / V_{E P}$ igual a 0.70). Para tal fin, se modificó el modelo analítico del eslabón para que no alcanzara su condición de falla, sin importar el nivel de deformaciones y rotaciones. En la figura 12 se muestran los ADI's del modelo 4N_MCE sometido a la acción de la secuencia $(E P+R)$ y su respectivo evento principal (EP), considerando y sin considerar la falla del eslabón (denominados CF y SF, respectivamente). Se puede observar que los valores de distorsión máxima de entrepiso de ambas modelaciones son idénticos hasta un nivel de intensidad de $1.2 \mathrm{~g}$. Sin embargo, el modelo que considera un criterio de falla en el eslabón exhibe mayores distorsiones máximas de entrepiso cuando es sujeto a la secuencia $\mathrm{EP}+\mathrm{R}$, mientras que los valores de la distorsión máxima de entrepiso crecen proporcionalmente al nivel de intensidad en el modelo sin criterio de falla. Inclusive, las distorsiones máximas de entrepiso son sensiblemente diferentes en los modelos CF y SF al ser sujetos al evento principal a partir de una intensidad de $1.9 g$. Por ello, es importante considerar el criterio de falla en la modelación de los eslabones de contraventeos excéntricos para poder examinar su comportamiento sísmico, principalmente ante secuencias sísmicas evento principal-réplicas. 


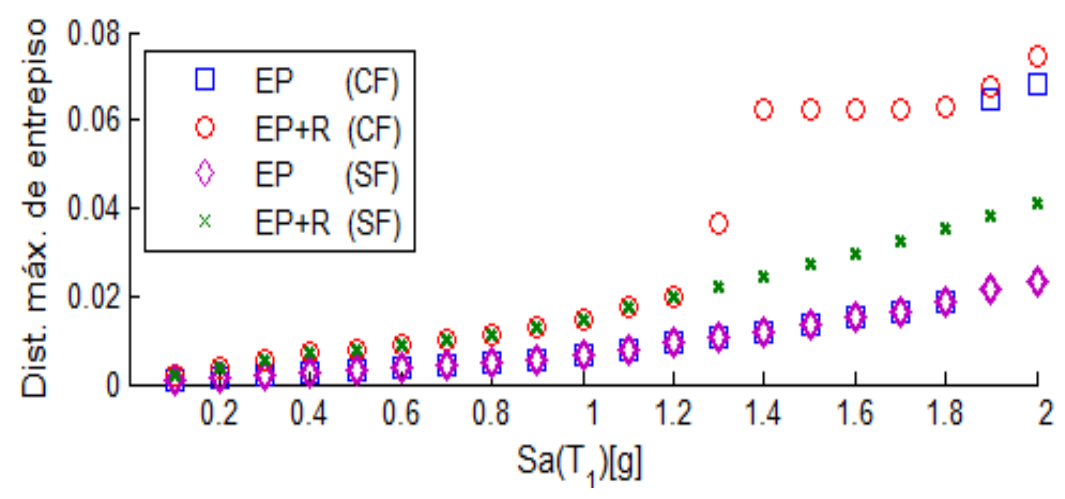

Figura 12. ADI del modelo 4N_MCE ante la secuencia EP2R6(70\%), considerando y sin considerar un cirterio de falla en el eslabón

Como se comentó en la Introducción de este trabajo, se espera que los eslabones disipen la energía inducida durante la excitación sísmica, por medio de su comportamiento histerético, mientras los restantes elementos estructurales (contraventeo, vigas, columnas) se diseñan para exhibir un comportamiento elástico. Por ello, resulta pertinente revisar esta suposición ante la acción de secuencias evento principal-réplicas. En la figura 13 se muestran las curvas histeréticas de los eslabones del modelo 4N_MCE ante la secuencia EP2R3(100\%) durante el evento principal y durante la réplica.
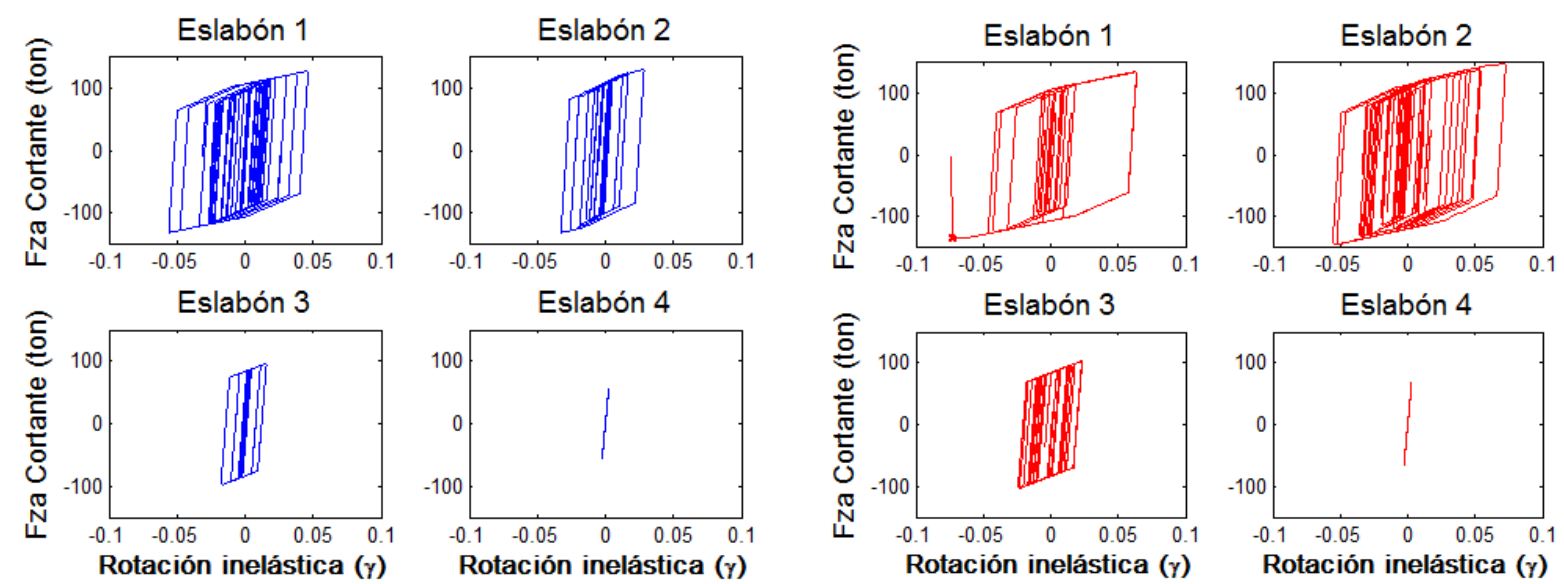

(a)

(b)

Figura 13. Curvas histeréticas de los eslabones del modelo 4N_MCE sometido a la secuencia sísmica EP2R3(100\%) para una intensidad de $0.8 g$ : a) ante el evento principal, b) ante la réplica

En la figura se puede observar que los eslabones de los primeros tres niveles exhiben un comportamiento inelástico ante la acción del evento principal, pero sin alcanzar el criterio de falla (figura 13a). Si los eslabones, principamente el del primer nivel, no son sujetos a un proceso de reparación (Gulec et al., 2011), su nivel de rotación inelástica, y plástica, puede incrementarse ante la acción de una réplica intensa como se ilustra en la figura 13b. En dicha figura se muestra que el eslabón del primer nivel alcanzó la rotación asociada al criterio de falla. Por otro lado, en la figura 14a se indica en el marco la ubicación del eslabón que ha fallado mientras que en la figura 14b se muestran las curvas histeréticas de los elementos estructurales del primer piso antes de que el eslabón alcance el criterio de falla (línea en color azul) y después de que alcanza el criterio de falla (línea en color rojo). Puede apreciarse que las vigas y columnas exhiben un comportamiento inelástico una vez que el eslabón alcanza el criterio de falla, lo cual es opuesto a su filosofía de diseño, mientras que los contraventeos permancen elásticos. 


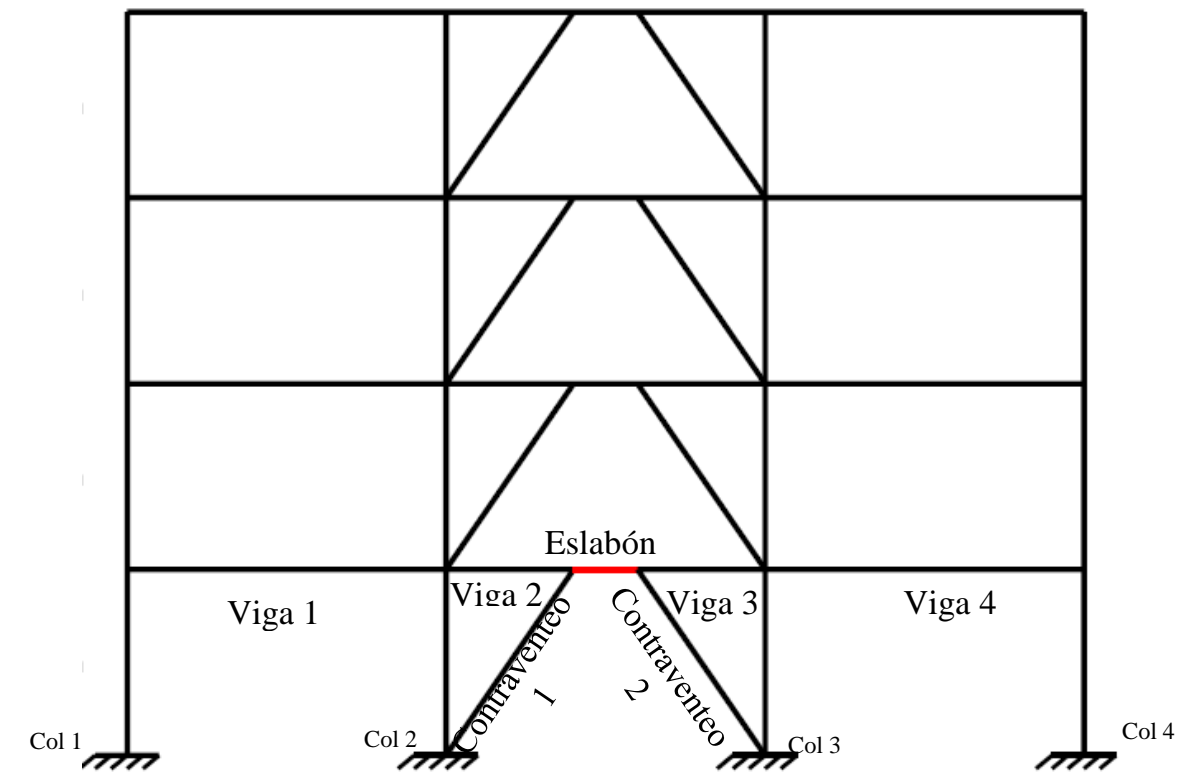

(a)
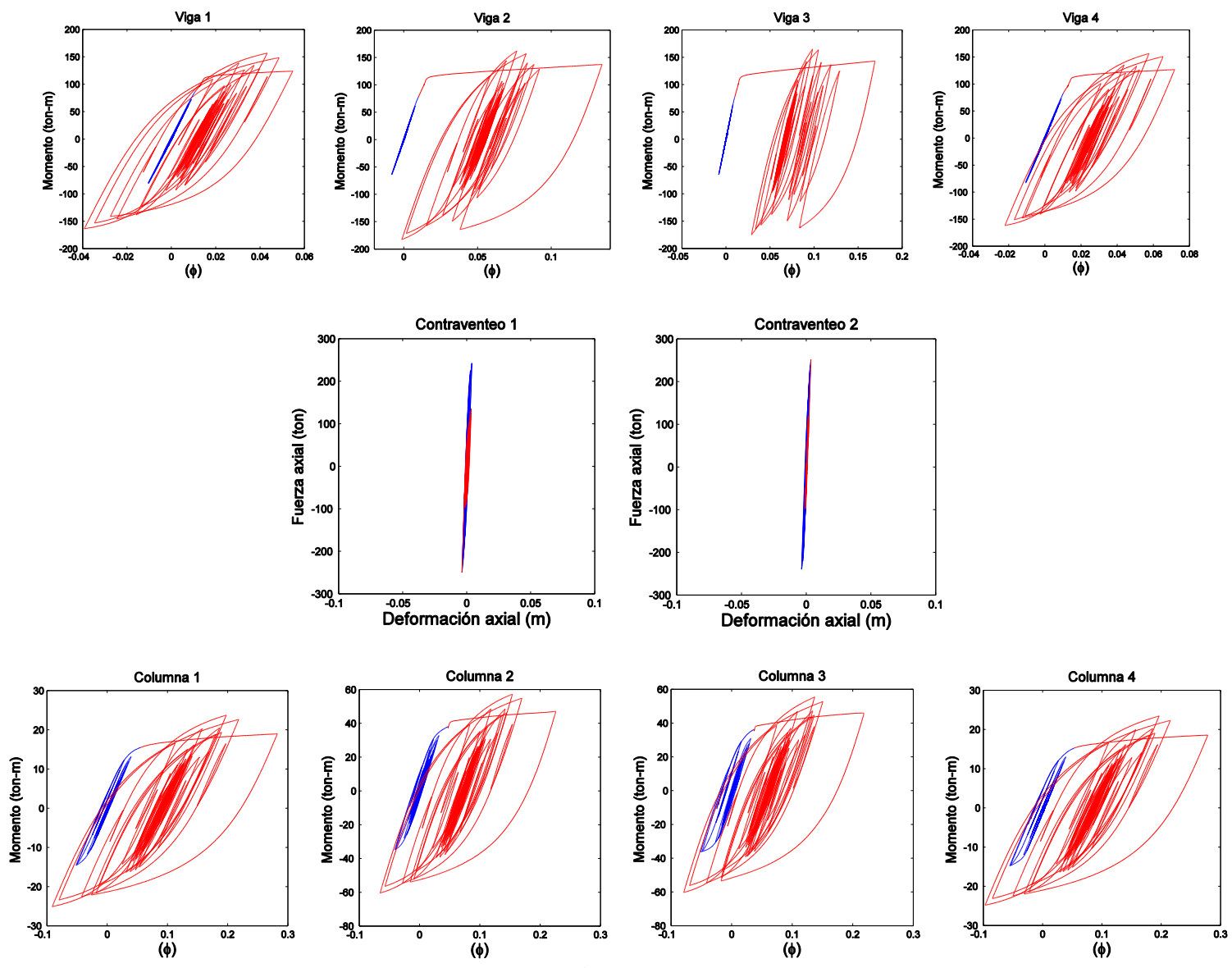

(b)

Figura 14. a) Ubicación del eslabón que alcanzó el criterio de falla en el modelo 4N_MCE ante la secuencia EP2R3(100\%) y b) curvas histeréticas de los elementos vigas y columnas cercanas al eslabón 


\section{Análisis dinámico incremental}

En las figuras 15 y 16 se ilustran los resultados del ADI en términos de distorsiones máximas de entrepiso del modelo 4N_MCE y del modelo 8N_MCE, respectivamente, sometidos a todas las secuencias del conjunto EP-R100\%. En la figura 15 se observa que los valores de distorsión máxima de entrepiso tienen una tendencia creciente lineal para los primeros 8 niveles de intensidad, a excepción de un punto en el nivel de intensidad $0.8 \mathrm{~g}$. Sin embargo, los valores de la distorsión máxima de entrepiso se incrementan considerablemente, respecto a la tendencia anterior, a partir de una intensidad de $0.9 \mathrm{~g}$ (es decir, conforme se incrementa el nivel de intensidad es mayor el número de datos que registran valores altos). En general, se pueden separar los resultados de los análisis en dos grupos. El grupo 1 corresponde a secuencias donde la distorsión máxima de entrepiso tiende a sufrir mayores incrementos a medida que crece la intensidad del movimiento del terreno, mientras que el grupo 2 corresponde a secuencias que no producen grandes incrementos de distorsión máxima de entrepiso, a pesar de los altos niveles de intensidad de las secuencias sísmicas. Una tendencia similar a la descrita anteriormente se puede observar para el modelo 8N_MCE salvo que la distinción de los dos grupos se puede identificar a partir de una intensidad de $1.1 \mathrm{~g}$.

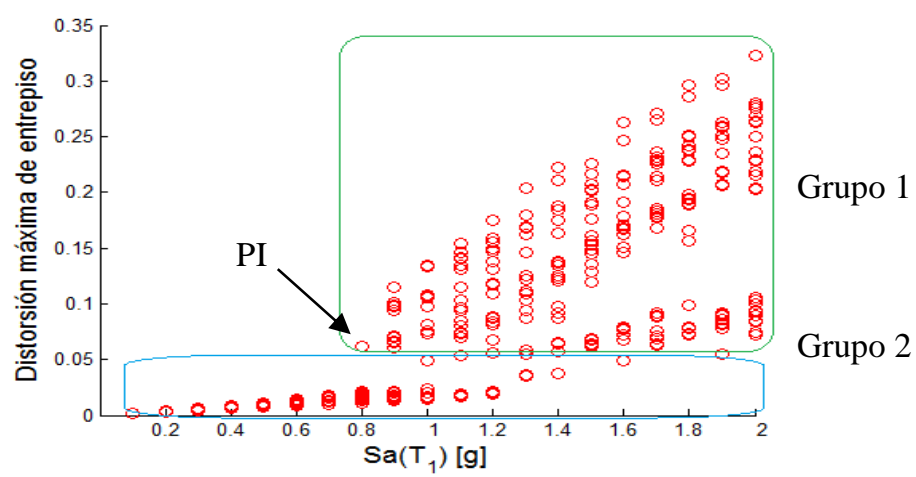

Figura 15. Resultados del ADI para el modelo 4N_MCE sometido a las secuencias sísmicas del grupo EP-R100\%

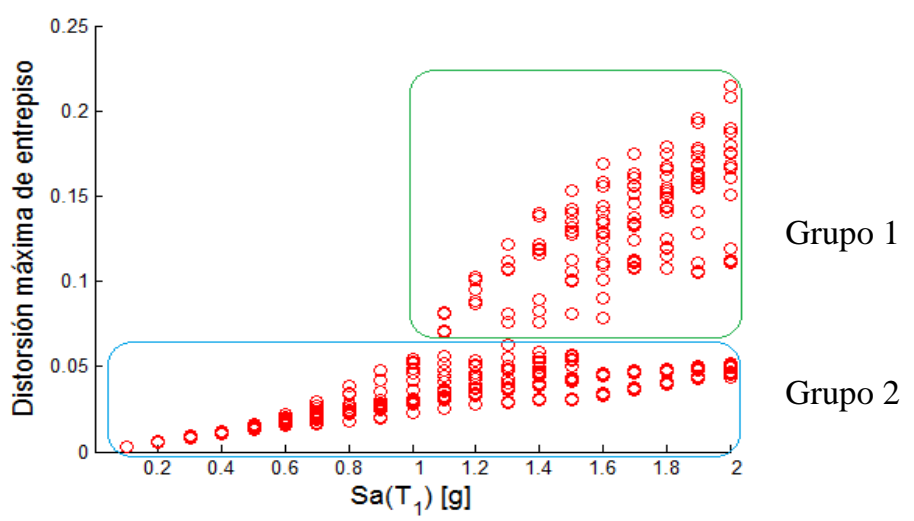

Figura 16. Resultados del ADI para el modelo 8N_MCE sometido a las secuencias sísmicas del grupo EP-R100\%

Para explicar los resultados obtenidos del grupo 1, se ha analizado el resultado aislado asociado a un nivel de intensidad sísmica de $0.8 \mathrm{~g}$ (punto PI en la figura 15). En la figura 17 se ilustran las curvas de comportamiento histerético fuerza cortante-rotación inelástica de los eslabones de cada entrepiso para el modelo 4N_MCE al ser sujeto a la secuencia EP2R3 (es decir, se utilizan los registros 2 y 3 de la tabla 2 como evento principal y réplica, respectivamente). En la figura se puede observar que los eslabones de los tres primeros entrepisos exhiben comportamiento inelástico, mientras que el eslabón del último entrepiso 
presenta un comportramiento elástico. En particular, el eslabón del primer entrepiso alcanzó una rotación igual a 0.06 , por lo que se consideró que falló y, en consecuencia, la falla de dicho eslabón provocó que las demandas de distorsión máxima se incrementaran en dicho entrepiso al final de la acción de la secuencia sísmica. Con la finalidad de verificar que el resto de las secuencias sísmicas escaladas a un nivel de intensidad de $0.8 \mathrm{~g}$ no producen la falla de algún eslabón en el marco, se trazaron las curvas histeréticas de los eslabones de cada entrepiso y se comprobó que ninguna de éstas había ocasionado que los eslabones fallarán. Lo anterior explica porqué en la figura 16 se tiene únicamente un dato con distorsion máxima de entrepiso excesivamente diferente para una intensidad de $0.8 g$. Finalmente, una inspección del comportamiento de los eslabones para niveles de intensidad superiores a $0.8 g$ reveló que las distorsiones máximas de entrepiso asociadas al grupo 1 se deben a que al menos uno de los eslabones ha fallado en el modelo ante las secuencias sísmicas.
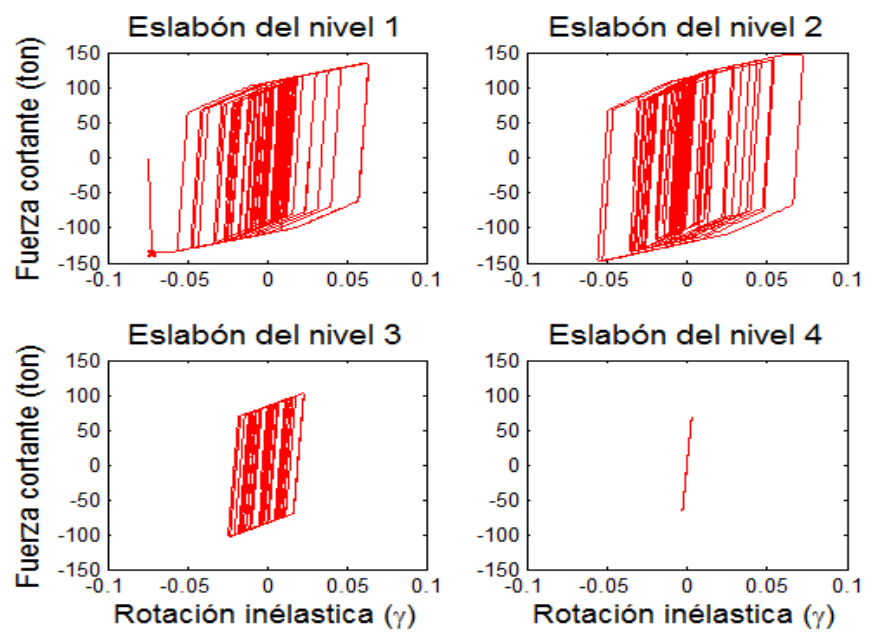

Figura 17. Respuesta histerética de los eslabones del modelo 4N_MCE ante la secuencia EP2R3(100\%), para un nivel de intensidad de $0.8 g$

En la figura 18 se muestran las curvas histeréticas fuerza cortante-rotación inelástica de los eslabones en el modelo 8N_MCE ante la secuencia sísmica EP1R5(100\%) escalada a un nivel de intensidad de 1.1 $g$. Se puede observar que los eslabones de cada entrepiso, a excepción del último, incursiona en el intervalo inelástico y, en particular, se observa que el eslabón del nivel 3 ha alcanzado el criterio de falla.

En general, puede decirse que los grandes incrementos en la distorsión máxima de entrepiso fueron causados por la falla de alguno de los eslabones. Por lo anterior, es de vital importancia el diseño cuidadoso tanto del eslabón como de los contraventeos, con la finalidad de minimizar la respuesta estructural de edificios ante secuencias sísmicas.

\section{Influencia de la intensidad sísmica}

En la figura 19 se muestran los perfiles de distorsión máxima de entrepiso (en línea gris) para el modelo 4N_MCE correspondientes a cuatro niveles de intensidad sísmica $(0.3 g, 0.7 g, 1.1 g$ y $1.5 g)$, así como la distorsión de entrepiso promedio (en línea azul) de las 28 secuencias sísmicas incluidas en el conjunto EP-R100\%. Se puede observar que la distorsión promedio máxima de entrepiso se incrementa sensiblemente de una intensidad de $0.7 \mathrm{~g}$ a $1.1 \mathrm{~g}$, y ésta tiende a concentrarse en los niveles inferiores para los niveles de intensidad de $1.1 \mathrm{~g}$ y $1.5 \mathrm{~g}$. Como ya se ha mencionado anteriormente, el incremento significativo en demandas de distorsión puede atribuirse a que los eslabones de los niveles inferiores alcazaron el criterio de falla. 

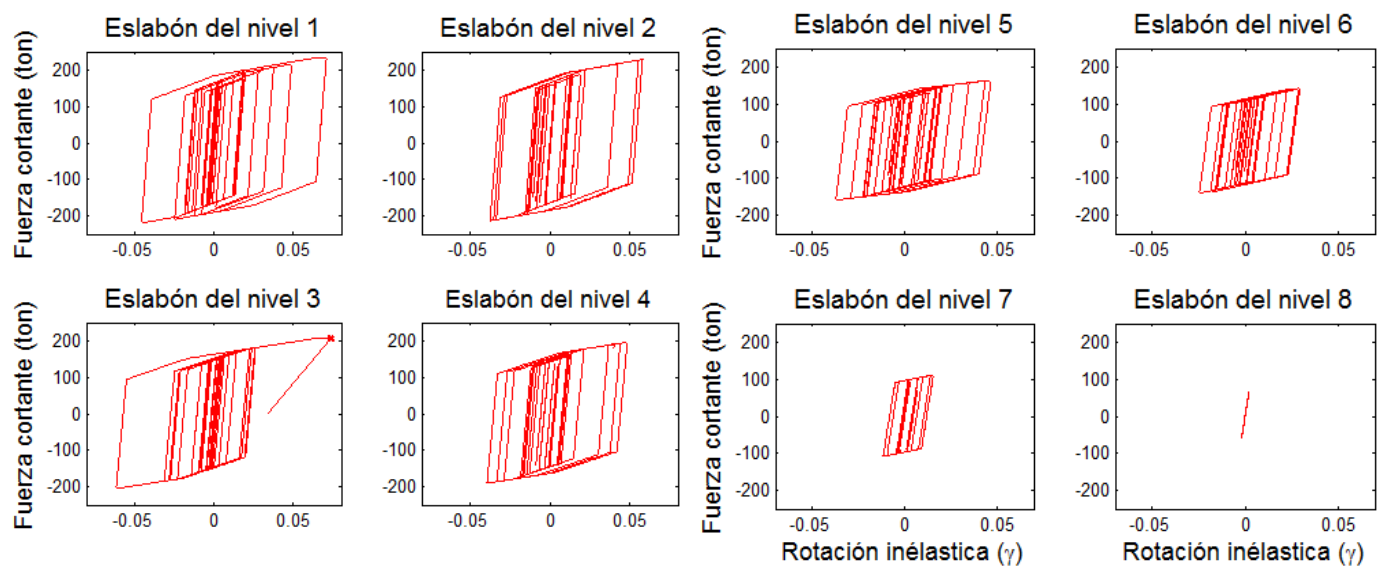

Figura 18. Curvas histeréticas de los eslabones, del modelo 8N_MCE ante la secuencia EP1R5(100\%), para un nivel de intensidad de $1.1 \mathrm{~g}$
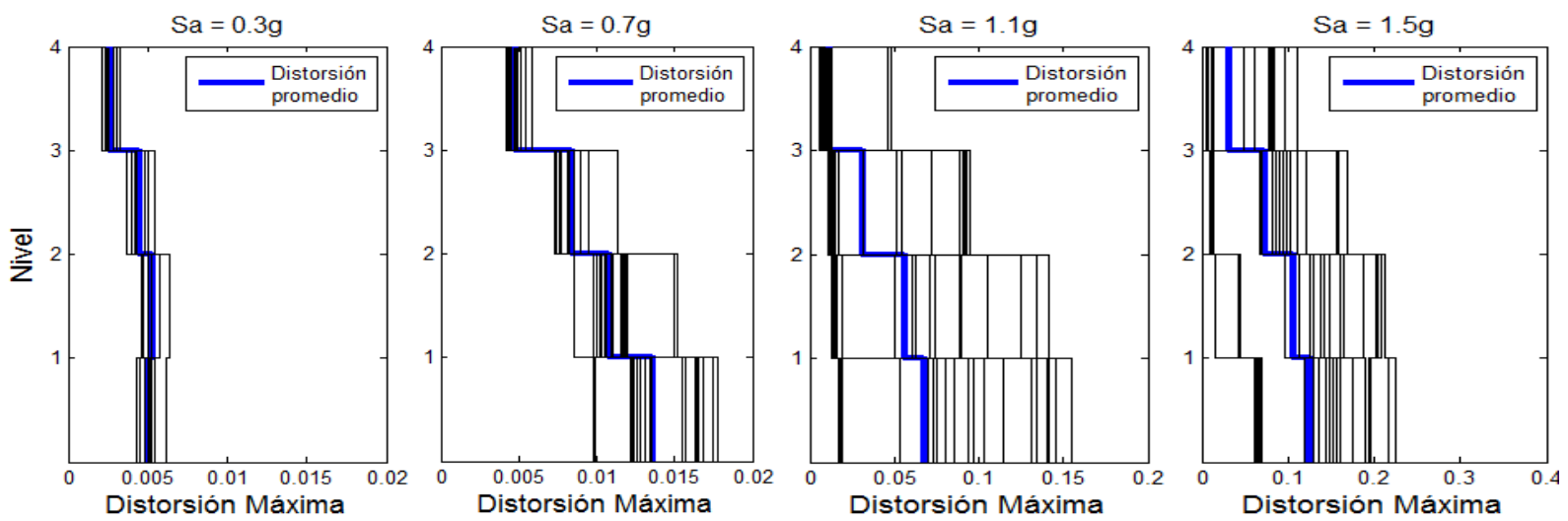

Figura 19. Perfiles de distorsión máxima del modelo 4N_MCE ante las secuencias sísmicas EP-R100\%

A continuación, en las figuras 20 y 21 se presentan los perfiles de distorsión máxima de entrepiso promedio correspondientes a tres niveles de intensidad $(0.5 g, 1.0 g$ y $1.5 g)$ para los modelos 4N_MCE y $8 \mathrm{~N} \_$MCE, respectivamente, considerando los tres conjuntos de secuencias sísmicas. Las líneas continuas consideran la respuesta únicamente ante el evento principal (SR) y las líneas discontinuas consideran la respuesta ante el evento principal y la réplica (CR). En la figura 20a se puede apreciar que la respuesta ante SR y CR es idéntica para los tres niveles de intensidad sísmica, lo que indica que las réplicas de las secuencias incluidas en el conjunto EP-R35\% no influyen en la respuesta del modelo 4N_MCE. Sin embargo, las distosiones máximas de entrepiso promedio sí se incrementan ante los conjuntos EP-R70\% y EP-R100\%. Cabe aclarar que el nivel de distorsiones ante las secuencias del grupo EP-R35\% son mayores que las distorsiones ante las secuencias de los grupos EP-R70\% y EP-R100\% dado que se emplearon factores de escala más grandes para alcanzar intensidades de $1.0 \mathrm{~g}$ y $1.5 \mathrm{~g}$. En particular, el incremento en las distorsiones asociadas a los niveles de intensidad de $1.0 \mathrm{~g}$ y $1.5 \mathrm{~g}$ ante las secuencias del grupo EP-R100\% son significativas, lo cual puede atribuirse a la falla de algún eslabón en los niveles inferiores del marco provocando que el modelo experimente grandes distorsiones laterales ante las réplicas mientras que las distorsiones de los niveles superiores son sensiblemente menores. 


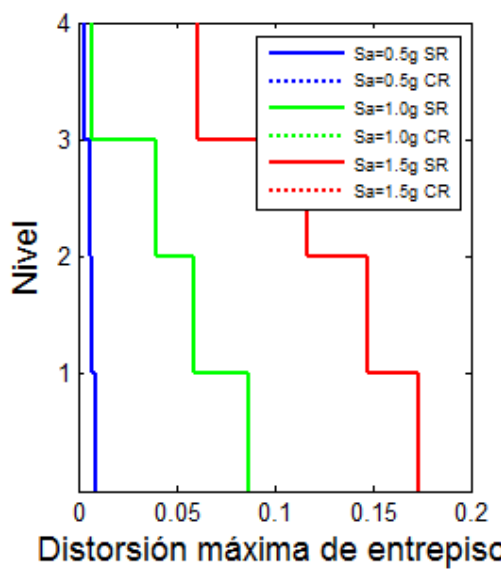

(a)

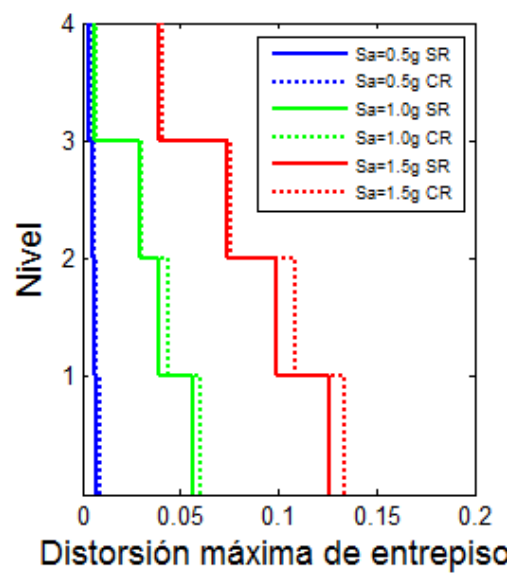

(b)

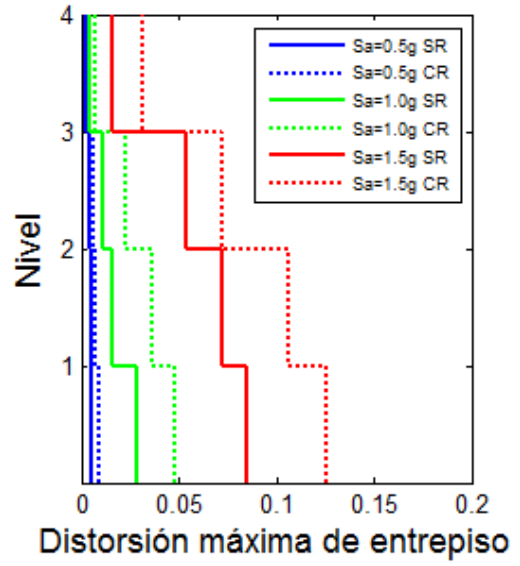

(c)

Figura 20. Perfil de distorsiones del modelo 4N_MCE, para tres niveles de intensidad: $0.5 g, 1.0 g, 1.5 g$, con y sin réplica, para los tres conjuntos de secuencia sísmica: a) conjunto EP-R35\%,

b) conjunto EP-R70\% y c) conjunto EP-R100\%

En la figura 21 se muestra una comparación similar correspondiente al modelo 8N_MCE, en la cual se puede apreciar una tendencia similar a la descrita para el modelo 4N_MCE. Es decir, la acción de las réplicas incluidas en el conjunto EP-R35\% no generan incrementos en la distorsión máxima de entrepiso, (no obstante que se obtienen distorsiones mayores que ante los conjuntos de secuencias EP-R70\% y EP$\mathrm{R} 100 \%$, dado que se emplearon factores de escala mayores para alcanzar intensidades de $1.0 \mathrm{~g}$ y $1.5 \mathrm{~g}$ ). Sin embargo, las réplicas tienden a incrementar la distorsión de entrepiso cuando se consideran los conjuntos de secuencias sísmicas EP-R70\% y, principalmente, EP-R100\%. Asimismo, es notoria la distribución nouniforme de distorsión de entrepiso en la altura del marco, principalmente cuando se incrementa el nivel de intensidad. Esta característica en la respuesta sugiere que los eslabones no tienen un comportamiento histerético uniforme en la altura (como se evidenció en las figuras 17 y 18), lo cual implica que su capacidad de disipación de energía no se aprovecha en su totalidad, principalmente en los eslabones de los pisos superiores.

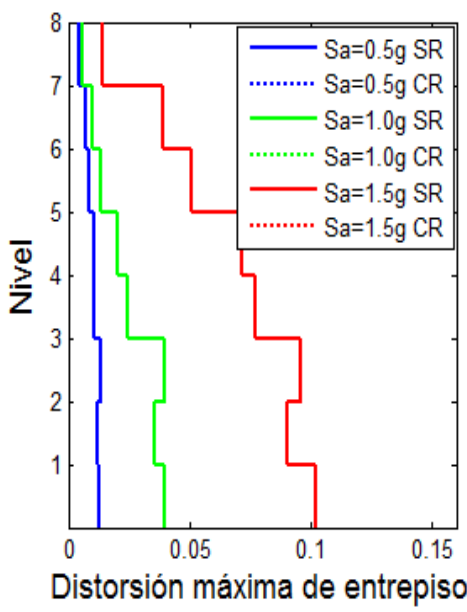

(a)

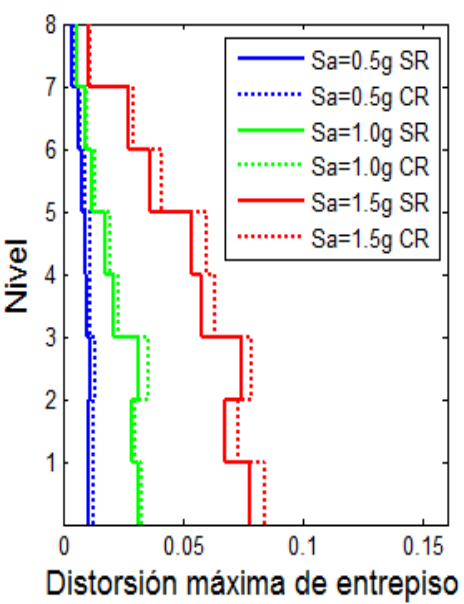

(b)

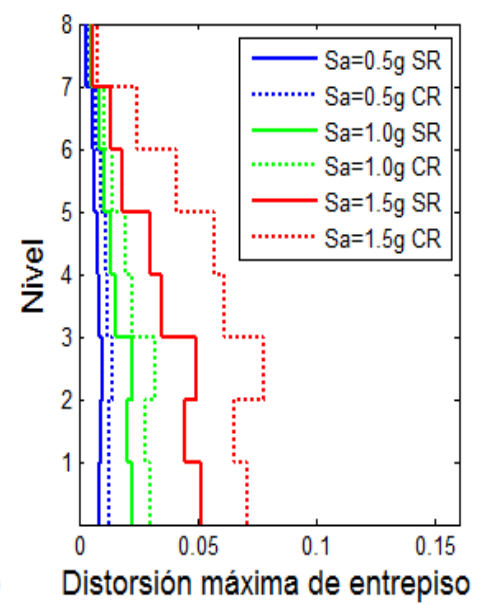

(c)

Figura 21. Perfil de distorsiones del modelo 8N_MCE, para tres niveles de intensidad: $0.5 g, 1.0 g, 1.5 g$, con y sin réplica, para los tres conjuntos de secuencias sísmicas: a) conjunto EP-100\%,

b) conjunto EP-R70\% y c) conjunto EP-R35\% 


\section{RESUMEN Y CONCLUSIONES}

Se estudió el comportamiento sísmico de marcos de acero de 4 y 8 niveles con contraventeos excéntricos (MCE) ubicados en terreno blando de la Ciudad de México. Los MCE fueron diseñados con base en la edición 2004 del Reglamento de Construcciones del Distrito Federal y sus correspondientes Normas Técnicas Complementarias para el Diseño Sísmico así como para el Diseño y Construcción de Estructuras Metálicas. Asimismo, se diseñaron los eslabones en los contraventeos excéntricos con base en las disposiciones del Instituto Americano de la Construcción en Acero (AISC) edición 2002. Los marcos en estudio se sometieron a un conjunto de secuencias sísmicas evento principal-réplica artificiales representativas de las secuencias registradas en terreno blando. Se consideraron tres niveles de intensidad de la réplica medida por el cociente $V_{R} / V_{E P}$, donde $V_{E P}$ es la velocidad máxima del terreno del evento principal escalada para alcanzar la velocidad máxima del terreno medida en la estación SCT durante el terremoto del 19 de septiembre de 1985 y $V_{R}$ es la velocidad máxima del terreno de la réplica. El desempeño sísmico de los marcos se evaluó mediante análisis dinámicos incrementales. Los resultados más relevantes obtenidos en esta investigación fueron los siguientes:

- La mediana de las demandas de distorsión máxima de entrepiso obtenida ante las secuencias sísmicas se incrementa, principalmente, con relaciones $V_{R} / V_{E P}$ iguales a 1.0 respecto a la mediana de las demandas de distorsión máxima de entrepiso obtenida ante el conjunto de eventos principales. Dicho incremento también depende del nivel de intensidad sísmica.

- La mediana de las demandas de distorsión máxima de entrepiso obtenida ante las secuencias sísmicas se incrementa, principalmente, con relaciones $V_{R} / V_{E P}$ iguales a 0.7 respecto a la mediana de las demandas de distorsión máxima de entrepiso obtenida ante el conjunto de eventos principales se incrementa ligeramente, y no existe incremento cuando la relación $V_{R} / V_{E P}$ iguales a 0.35 .

- Una vez que los eslabones alcanzaron el criterio de falla, generalmente ante la acción de las réplicas, las vigas y columnas que rodean los eslabones se comportaron nolinealmente, lo cual es opuesto a su filosofía de diseño que asume un comportamiento elástico ante la acción sísmica. Como consecuencia, las demandas de distorsión máxima de entrepiso se incrementan significativamente.

- Se observó una distribución no uniforme de la capacidad de disipación de energía por comportamiento cíclico (energía histerética) de los eslabones en la altura, lo cual condujo a una concentración de distorsión de entrepiso en los pisos inferiores.

Con base en los resultados obtenidos, se sugiere prestar especial cuidado al detallado (por ejemplo, soldado de los atiesadores en la zona del eslabón, conexión del contraventeo a eslabón) de los eslabones para fomentar que desarrollen niveles de rotación plástica sin exhibir un comportamiento histerético degradante prematuro. Asimismo, se recomienda proponer e investigar una metodología que optimice la distribución de la energía histerética de los contraventeos excéntricos, con la finalidad de aprovechar de mejor manera la capacidad de disipación de energía en los eslabones y evitar concentraciones de distorsión de entrepiso.

\section{AGRADECIMIENTOS}

Este trabajo se desarrolló con el apoyo económico brindado por el Consejo Nacional de Ciencia y Tecnología, CONACYT, a través del Proyecto CB-2011-01-167419, y la beca de maestría otorgada al tercer autor. Se agradece el apoyo de la Universidad Autónoma de Sinaloa dentro del proyecto PROFAPI 2015 y de la Universidad Michoacana de San Nicolás de Hidalgo. 


\section{REFERENCIAS}

AISC (2002) Seismic provisions for structural steel buildings, ANSI/AISC 341-02, American Institute of Steel Construction, Chicago, Illinois, 309 pp.

AISC (2016) Seismic provisions for structural steel buildings, ANSI/AISC 341-16, American Institute of Steel Construction, Chicago, Illinois, 309 pp.

ASCE (1990) Minimum design loads for buildings and other structures, ASCE 7-88, American Society of Civil Engineers, New York, New York, 94 pp.

Azad, S K y C Topkaya (2017), "A review of research on steel eccentrically braced frames", Journal of Constructional Steel Research, Vol. 128, pp. 53-73. DOI: 10.1016/j.jcsr.2016.07.032

Díaz, M A (2006), “Confiabilidad sísmica de edificios de acero diseñados con el RCDF-2004”, Tesis de Maestría, Facultad de Ingeniería, Universidad Nacional Autónoma de México, 69 pp.

Díaz-Martínez, G, J Ruiz-García y A Terán-Gilmore (2014), "Response of structures to seismic sequences corresponding to Mexican soft soils", Earthquakes and Structures, Vol. 7, pp. 1241-1258. DOI: 10.12989/eas.2014.7.6.1241

Guerrero, H, J Ruiz-García, J A Escobar y A Terán-Gilmore (2017), "Response to seismic sequences of short-period structures equipped with buckling-restrained braces located on the lakebed zone of Mexico City", Journal of Constructional Steel Research, Vol. 137, pp. 37-51. DOI: 10.1016/j.jcsr.2017.06.010

Gulec, C K, B Gibbons, A Chen y A S Whittaker (2011), "Damage states and fragility functions for link beams in eccentrically braced frames", Journal of Constructional Steel Research, Vol. 67, No. 9, pp. 1299-1309. DOI: 10.1016/j.jcsr.2011.03.014

Gupta, A y H Krawinkler (1999), "Seismic demands for performance evaluation of steel moment resisting frame structures," Technical Report 132, The John A. Blume Earthquake Engineering Research Center, Department of Civil Engineering, Stanford University, Stanford, CA.

Kanvinde, A M, K S Marshall, D A Grilli y G Bomba (2014), "Forensic analysis of link fractures in eccentrically braced frames during the February 2011 Christchurch Earthquake - Testing and Simulation", Journal of Structural Engineering ASCE, 141(5). DOI: 10.1061/(ASCE)ST.1943541X.0001043

McKenna, F, G L Fenves, M H Scott y B Jeremic (2000), Open system for earthquake engineering simulation (OpenSees). Pacific Earthquake Engineering Research Center, University of California, Berkeley.

Meli, R y J A Avila (1989), "The Mexico earthquake of September 19, 1985-Analysis of building response", Earthquake Spectra, Vol. 5, No. 1, pp. 1-18. DOI: 10.1193/1.1585506

Miranda, E (1993), "Evaluation of site-dependent inelastic seismic design spectra", Journal of Structural Engineering, ASCE, 119(5): pp. 1319-1338. DOI: 10.1061/(ASCE)0733-9445(1993)119:5(1319)

NTC-A (2004), "Normas técnicas complementarias para el diseño y construcción de estructuras de acero", Gaceta Oficial del Gobierno del Distrito Federal, agosto.

NTC-A (2017), "Normas técnicas complementarias para el diseño y construcción de estructuras de acero", Gaceta Oficial del Gobierno de la Ciudad de México, diciembre.

Okazaki, T, G Arce, H-C Ryu y M D Engelhardt (2005), "Experimental study of local buckling, overstrength, and fracture of links in eccentrically braced frames", Journal of Structural Engineering, 131(10), pp. 1526-35. DOI: 10.1061/(ASCE)0733-9445(2005)131:10(1526) 
Prinz, G S (2010), “Using buckling-restrained braces in eccentric configurations", PhD. Dissertation, Brigham Young University. https://scholarsarchive.byu.edu/etd/2134

Ramadan, T y A Ghobarah (1995), "Analytical model for shear-link behavior", Journal of Structural Engineering, ASCE, Vol. 121, No.11, pp. 1574-1580. DOI: 10.1061/(ASCE)07339445(1995)121:11(1574)

Richards, P W (2004), "Cyclic stability and capacity design of steel eccentrically braced frames" Ph.D. dissertation, Dept. of Structural Engineering, Univ. of California, San Diego, La Jolla, CA.

Rosenblueth, E y R Meli, (1986), “The 1985 Mexico earthquake: causes and effects in Mexico City”, Concrete International, ACI, Vol. 8, No. 5, pp. 23-34.

Ruiz-García J (2012), "Mainshock-aftershock ground motion features and their influence in building's seismic response", Journal of Earthquake Engineering, Vol. 16, No. 5, pp. 719-737. DOI: $\underline{10.1080 / 13632469.2012 .663154}$

Ruiz-García J, M V Marín y A Terán-Gilmore (2014), "Effect of seismic sequences in reinforced concrete frame buildings located in soft-soil sites", Soil Dynamics and Earthquake Engineering, Vol. 63, pp. 56-68. DOI: 10.1016/j.soildyn.2014.03.008

Tapia, E y A Tena (2011), "Factores de ductilidad y sobrerresistencia en marcos de acero con contraventeo chevron", Revista de Ingeniería Sísmica, Vol. 84, pp. 47-68. DOI: 10.18867/ris.84.25

Terán-Gilmore, A, A Sanchez-Badillo, M Espinosa-Johnson (2010), "Performance-based seismic design of reinforced concrete ductile buildings subjected to large energy demands", Earthquakes and Structures; Vol. 1, No. 1, pp. 69-92. DOI: 10.12989/eas.2010.1.1.069

Vamvatsikos, D y C A Cornell (2002), "Incremental dynamic analysis", Earthq Eng. Struct Dynam; Vol. 31, No. 3, pp. 491-514. DOI: 10.1002/eqe.141 